Revue musicale OICRM

\title{
Musique et immersion spectatorielle dans les scènes de poursuite des films d'action du cinéma hollywoodien contemporain
}

\section{Hubert Bolduc-Cloutier}

Volume 5, numéro 2, 2018

Création musicale et sonore dans les blockbusters de Remote Control

URI : https://id.erudit.org/iderudit/1054147ar

DOI : https://doi.org/10.7202/1054147ar

Aller au sommaire du numéro

Éditeur(s)

Observatoire interdisciplinaire de création et recherche en musique (OICRM)

ISSN

2368-7061 (numérique)

Découvrir la revue

Citer cet article

Bolduc-Cloutier, H. (2018). Musique et immersion spectatorielle dans les scènes de poursuite des films d'action du cinéma hollywoodien contemporain. Revue musicale OICRM, 5(2), 48-78. https://doi.org/10.7202/1054147ar
Résumé de l'article

En tant qu'un des principaux types de scène des films d'action, la poursuite constitue un point culminant de la narrativité filmique au sein duquel la réponse sensorielle du spectateur atteint son paroxysme. Afin de considérer quels sont les agents musicosonores de l'immersion spectatorielle dans les scènes de poursuite du cinéma hollywoodien contemporain et la structure rhétorique de ce type de scène, cet article propose une approche au carrefour des recherches sur l'immersion spectatorielle au cinéma (Barker), l'immersion dans les musiques de jeux vidéo (Grimshaw, Calleja), de même que la création musicale dans le cadre de l'Electronic Dance Music (Butler). Les caractéristiques musicales récurrentes et prégnantes sont ici soulignées pour tracer le portrait de l'esthétique sonore des scènes de poursuite réalisées par les compositeurs affiliés à Remote Control. 


\title{
Musique et immersion spectatorielle dans les scènes de poursuite des films d'action du cinéma hollywoodien contemporain
}

\author{
Hubert Bolduc-Cloutier
}

\begin{abstract}
Résumé
En tant qu'un des principaux types de scène des films d'action, la poursuite constitue un point culminant de la narrativité filmique au sein duquel la réponse sensorielle $\mathrm{du}$ spectateur atteint son paroxysme. Afin de considérer quels sont les agents musicosonores de l'immersion spectatorielle dans les scènes de poursuite du cinéma hollywoodien contemporain et la structure rhétorique de ce type de scène, cet article propose une approche au carrefour des recherches sur l'immersion spectatorielle au cinéma (Barker), l'immersion dans les musiques de jeux vidéo (Grimshaw, Calleja), de même que la création musicale dans le cadre de l'Electronic Dance Music (Butler). Les caractéristiques musicales récurrentes et prégnantes sont ici soulignées pour tracer le portrait de l'esthétique sonore des scènes de poursuite réalisées par les compositeurs affiliés à Remote Control.
\end{abstract}

Mots clés : musique de film ; cinéma ; scènes de poursuite ; immersion ; Remote Control.

\begin{abstract}
As one of the main action movies type of scenes, the chase is a peak where cinema-goers sensorial response reaches a climax. In order to consider sonic and musical stimulators of immersion in contemporary Hollywood cinema chasing scenes and to evaluate the rhetoric of this type of scenes, this article proposes an approach at the crossroads of research in spectatorial immersion in cinema (Barker), immersion in video games music (Grimshaw, Calleja), as well as musical creation in Electronic Dance Music (Butler). Recurring and significant musical characteristics are highlighted to chart the sound aesthetic profile of chasing scenes conceived by composers affiliated with Remote Control.
\end{abstract}

Keywords: film music; cinema; chase scenes; immersion; Remote Control. 
Déferlement vertigineux d'images et de sons, les scènes de poursuite constituent des points culminants de la narrativité filmique au sein desquels la réponse sensorielle du spectateur atteint son paroxysme. Depuis l'avènement de l'ère numérique et notamment de l'esthétique sonore prônée par le studio Remote Control sous la direction de Hans Zimmer, la musique de film hollywoodienne se montre sous des aspects variés et novateurs, proposant une mutation de l'écriture orchestrale du néoclassicisme ${ }^{1}$, et intégrant de nouvelles ressources soniques destinées à intensifier l'expérience sensible du film. Les principales caractéristiques de cette esthétique sonore ont un écho particulier dans le cadre de la poursuite où elles sont mis en exergue, concentrées en une période restreinte, et offrent des moyens pertinents pour répondre aux contraintes inhérentes à ce type de scène. Alors que la structure narrative de la poursuite a fait l'objet de plusieurs études pour les cinémas primitif et muet (Burch 1983 ; Auerbach 2000 ; McCaffrey 2016), la dimension sonore et plus spécifiquement la musique des scènes de poursuite ne semblent pas avoir été traitées de front dans la littérature, mais plutôt abordées au sein du profil général des scènes d'action ou encore des films d'horreur (Donnelly 2009 ; Lerner 2010). Par ailleurs, l'unique étude menée à notre connaissance sur les relations entre le corps du spectateur et le film dans le processus immersif des scènes de poursuite néglige les éléments sonores de la cinématographie (Barker 2009). Au carrefour de ces différentes approches, nous entendons considérer quels sont les composants structurants des scènes de poursuite du cinéma hollywoodien contemporain, en portant une attention particulière aux agents musicosonores qui participent à l'immersion spectatorielle.

À partir de notre corpus de scènes issues de blockbusters des années 2010 et dont les musiques ont été réalisées par des compositeurs associés à Remote Control ${ }^{2}$, nous chercherons à définir les éléments déterminants de chaque partie de la structure rhétorique de la poursuite, en soulignant l'importance du rôle joué par le rythme et la texture sonore. De même, nous verrons que l'esthétique immersive et spectaculaire qui définit l'ensemble sonore de la poursuite partage d'une part des propriétés communes à d'autres types de musiques créées à partir d'outils numériques (notamment 1'Electronic Dance Music), et rejoint, d'autre part, les réflexions développées autour des qualités

1 Il est question ici du néoclassicisme hollywoodien au sens où l'entend, par exemple, Emilio Audissino (Audissino 2014).

2 La liste des compositeurs associés, ou ayant été associés, à Remote Control est présentée dans l'onglet «Team (Present \& Past ) » sur le site officiel de Hans Zimmer. Les scènes mises à l'étude ont été sélectionnées en fonction des pistes comportant le terme «chase » dans la liste des albums de films d'action ayant eu une publication officielle entre 2010 et 2017 inclusivement. De plus, l'ultime scène de poursuite de Mad Max. Fury Road (2015) qui a servi de base à notre projet de recherche a été intégrée au corpus, de même que la scène de poursuite en voiture du film Ninja Turtles 2 (Green, 2016, cue "Shredder Escape "). Ainsi établi, notre corpus comprend 18 scènes de poursuite tirées de 14 productions filmiques. Notre corpus ne comprend pas les pistes associées aux jeux vidéo numériques, aux séries télévisées, ni aux productions françaises. Enfin, la piste " Note Chase » de l'album du film The Sorcerer's Apprentice (2010) a été écartée comme étant non pertinente par rapport aux autres pistes du corpus, mettant en scène la poursuite d'un objet. À noter que la liste des 62 compositeurs présentés sur le site de Hans Zimmer ne comporte qu'une seule femme, Shirley Walker (1945-2006), et aucun Afro-Américain. Voir le corpus en annexes. 
immersives des jeux vidéo numériques. Enfin, soulignons que les différentes caractéristiques musicales soulevées dans notre étude ne sont pas systématiques, interviennent à différents degrés, et peuvent également se retrouver dans d'autres types de scènes d'action. De plus, notre intérêt porte davantage sur les propriétés musicales récurrentes des scènes de poursuite de notre corpus, sans mettre en évidence les particularités esthétiques des compositeurs abordés ${ }^{3}$.

\section{LOGIQUE NARRATIVE DES SCÈNES DE POURSUITE}

Le principe du climax inhérent à la scène de poursuite implique une augmentation sensible et constante de la tension jusqu'à un point d'apogée qui, en fonction du dénouement de la poursuite, va marquer un tournant dans le développement narratif. Elle constitue l'un des trois principaux types de scènes d'action avec « la bagarre ou le combat, la lutte contre un mécanisme et l'accident ${ }^{4}$ "(Arijon 2004, p. 483). La poursuite permet de plus un renforcement de l'empathie du spectateur envers le héros en posant ce dernier dans un contexte périlleux au sein duquel s'affirme le rapport dichotomique entre la figure vertueuse qu'il incarne et ses rivaux. En ce sens, la scène de poursuite est un procédé narratif filmique qui répond de manière particulièrement efficace à l'un des principes fondamentaux de la construction classique hollywoodienne, soit l'identification aux personnages. Comme 1'affirme David Bordwell :

The readiest way to make us care about characters is to plunge them into a swirl of cause and effect, goals and obstacles, conflicts and resolutions, appointments and deadlines, patterns of restricted and unrestricted narration, recurring motifs and dangling causes and symmetrical closure - in short, all the resources of tradition (Bordwell 2006, p. 107).

Alors que l'issue de la poursuite affecte le développement narratif, le corps de la scène constitue une suspension dans la narration d'une durée variable ayant une forte propension au spectaculaire. De telle manière, il est cohérent que la poursuite soit intégrée dans une majorité de films d'action hollywoodiens afin d'exploiter une large variété d'effets spéciaux et répondre à la « culture de la stimulation » (Gunning 2006, p. 65) inhérente au cinéma des attractions tel que décrit par Tom Gunning. Ce dernier souligne d'ailleurs l'importance du film de poursuite dans le cinéma primitif comme "premier véritable genre narratif du cinéma, dans lequel [la poursuite] avait introduit

\footnotetext{
3 Notre attention porte sur l'esthétique musicale globale de Remote Control qui présente une forte homogénéité, et ce notamment en fonction du mode de production mis en place sous l'égide de Hans Zimmer : "Zimmer has built considerable clout for the [Remote Control Production] brand whilst at the same time divesting responsibilities and delegating much of actual composing and producing. The financial benefits of centralizing operations and maintaining a relatively homogenous work-flow and output are manifold. Studio facilities can be shared and usage optimized, project acquisition can be streamlined, and multiple projects can be run concurrently. Although the underlying process may vary in the details-and while a specific director may work with one RCP composer rather than another-production value, reliability, and quality control are assured» (Hexel 2016, p. 6-7).

4 Nos références à l'ouvrage d'Arijon sont motivées par les qualités synthétiques des écrits de l'auteur sur l'esthétique des productions filmiques hollywoodiennes post-âge d'or. Aussi, selon David Bordwell : "Daniel Arijon's Grammar of the Film Language, a manual which professional directors sometimes consult in planning a scene, is virtually a compendium of the emerging staging and cutting styles " (Bordwell 2002, p. 21).
} 
un modèle de causalité et de linéarité, ainsi que les bases d'un montage reposant sur la continuité » (ibid., p. 63). De plus, l'intérêt de ce type de scène réside essentiellement dans l'expression du mouvement, dans la mise en valeur des propriétés de la cinématographie permettant de créer la temporalité, et, plus précisément, dans le jeu entre les polarités de la cadence kinésique auquel participent les éléments de la sémiotique filmique. De fait, la poursuite est fondée sur le besoin d'agir et de se déplacer, besoin qui devient également le « projet du spectateur » (Barker 2009, p. 109)à travers la mise en place de procédés cinématographiques immersifs.

\section{AgENTS DE L'IMMERSION SPECTATORIELLE}

Bien que Jennifer Barker omette le rôle joué par la dimension sonore dans le processus immersif des scènes de poursuite, elle soulève néanmoins nombre de spécificités quant à leur esthétique visuelle (ibid. ${ }^{5}$ ). À titre d'exemple, on retrouve le montage rapide et le recours à la caméra subjective "from within speeding vehicles in order to intensify in the muscular register the audience's sense of involvment, which is already encouraged by the narrative » (ibid., p. 111). Barker souligne le choix du placement de la caméra qui suscite chez le spectateur une volonté d'atteinte du but commune à celle du héros. Les points de vue du conducteur, du passager arrière, les plans de l'intérieur et de l'extérieur du véhicule sont fréquents dans les scènes de poursuite, tout comme les gros plans, les plans rapprochés sur des éléments clés, les cadres refermés et les longues focales ${ }^{6}$. Toutes techniques permettant de hausser et défamiliariser la perception quotidienne et provoquer la tension nerveuse sont employées pour favoriser l'immersion ${ }^{7}$.

Ces observations rejoignent les différentes manières de mettre en valeur une action visuelle dans le contexte des films d'action soulevées par Arijon. Ce dernier précise que

la combinaison de points de vue de la caméra, fixes et en mouvement apporte un meilleur impact visuel à une scène de poursuite ou à un groupe de gens en train de courir. Les vues les plus rapprochées des sujets sont faites à partir de positions fixes de caméra. Un plan statique est suivi d'un plan en travelling et la formule est constamment répétée avec éventuellement des pauses pendant l'action (Arijon 2004, p. 492).

Arijon ajoute par ailleurs que «les plans en mouvement peuvent parfois être saccadés (caméra à la main) [et] doivent donc être de courte durée (pas plus de cinq secondes environ), pour ne pas déranger l'assistance. Ce mouvement inégal ajoutera le plus

5 Voir plus précisément la partie « Apprehension » du chapitre 2, p. 106-119.

6 Ces propriétés visuelles des scènes de poursuite sont soulignées par Barker (Barker 2009, p. 113), qui abonde dans le sens des recherches antérieures de Bordwell (Bordwell 2002).

$7 \quad$ Cette approche est déjà présente dans les films de poursuite du cinéma primitif. Comme l'affirme Auerbach : "The chase's recursive pattern more generally derives from a certain hysterical formation, I have begun to suggest, that centers on anxieties about the human body " (Auerbach 2000, p. 810). Le film The Escaped Lunatic (Wallace McCutcheon, 1903) constitue en ce sens un exemple phare de la structure des films de poursuite du cinéma primitif. 
souvent un peu d'excitation à la poursuite » (Arijon 2004, p. 493). En résumé, les agents immersifs attribués à la dimension visuelle de la poursuite et auxquels on peut proposer des équivalences sonores sont : la vitesse du montage, un jeu d'alternance entre des pôles extrêmes, soit entre général (plan d'ensemble) et singulier (gros plan), ou entre mouvement rapide et statisme, ainsi que des techniques pour favoriser l'empathie (comme la caméra subjective).

Pour prolonger ce portrait initial, les réflexions développées autour des agents générateurs de l'immersion dans le contexte des jeux vidéo numériques offrent des correspondances pertinentes lorsqu'elles sont adaptées aux films hollywoodiens. Parmi celles-ci, on retient le modèle d'engagement constitué de sept cadres interrelationnels qui favorisent l'implication du joueur ( Digital Game Involvement Model ») proposé par Gordon Calleja (Calleja 2007) ${ }^{8}$, de même que le survol des études sur le sujet proposé par Mark Grimshaw (Grimshaw 2012). De l'ensemble des cadres de Calleja, quatre peuvent être, dans une certaine mesure, transposés au contexte filmique. D'abord, le cadre de l'engagement affectif correspond à l'attitude du joueur face au jeu. Le joueur a une certaine prédisposition en fonction du type de jeu abordé et s'attend à ce que ce dernier soit convaincant (" compelling »). Dans le contexte cinématographique, le spectateur connaît de façon active ou passive les codes du film d'action, l'aspect sonique du genre, voire même parfois la signature musicale d'un film en particulier (par exemple les séries James Bond, Mission Impossible, Die Hard, etc.). Ce premier cadre comporte un jeu entre la réponse aux attentes du spectateur face au genre abordé et des effets de surprise qui vont le défamiliariser et susciter la tension.

L'engagement narratif correspond à la position du joueur face à l'histoire d'un jeu, d'une mission ou d'une quête, et son interprétation de son expérience personnelle du jeu. Ce type d'engagement est toutefois différent dans le contexte cinématographique. Comme l'indique Calleja :

Players rarely have the same designed narrative expectations of games and MMOGs [massively multiplayer online games] as they do of literary works or movies, for example. Games emphasize player performance, while literary works and movies create compelling media experiences through their assemblage of form and content. The designed narrative and personal narratives in literary works and movies are far more closely aligned than in digital games (Calleja 2007, p. 87).

Néanmoins, on peut associer l'engagement narratif propre au jeu à celui de la narrativité filmique, et plus précisément à l'empathie envers les protagonistes qui permet de hausser l'implication émotionnelle du spectateur.

Ensuite, l'engagement spatial situe le joueur dans un espace plus large que celui présenté par l'écran du jeu. Il peut prendre la forme de représentation mentale de cartes, ou cartographie, où l'on perçoit les déplacements des autres joueurs et les distances qui peuvent éventuellement être parcourues. La représentation d'un environnement plus large que celui dans lequel se trouve le joueur donne un sens de

8 Les sept cadres de l'engagement de Calleja sont : tactile, performatif, affectif, partagé, narratif, spatial, et l'incorporation (qui correspond à l'immersion). 
grandeur qui contribue à rendre le monde du jeu plus réaliste. Ce genre de représentation est, nous le verrons, commun dans les scènes de poursuite.

Enfin, l'immersion sensitive - ou l'incorporation - résulte de la synthèse des autres cadres d'engagement et "operates on a double metaphor: incorporating (in the sense of assimilation or internalization) the environment while re-incorporating (in the sense of corporeal embodiment) the playerthrough the avatar in that evironment "(Calleja 2007, p. 88). Le contexte filmique étant non-ergodique (pas de contrôle ou de geste pour agir concrètement dans le film), et non-performatif (le spectateur ne contrôle pas un avatar qui agit dans le film), seule la première proposition, soit l'immersion sensitive sans réincorporation, peut y être transposée. Parmi les conditions générales pour favoriser l'état immersif soulevées par Calleja et dans l'article de synthèse de Grimshaw, il semble que la primauté de la sensation sur la réflexion soit centrale, au même titre que la réduction des efforts de conscience. Ainsi, la suspension du développement de la narrativité filmique pendant le corps de la poursuite et la mise en place de techniques, comme le montage rapide, qui contraignent le raisonnement cognitif au profit de réponses sensorielles relevant de l'automatisme chez le spectateur paraissent essentielles à la rhétorique de ce type de scène.

En somme, les éléments récurrents des discours abordés sur l'immersion dans les scènes de poursuite et dans les jeux numériques relèvent de l'engagement affectif et narratif (émotionnel), l'engagement temporel, l'engagement spatial, d'une diminution de la distance critique en prônant la sensation sur la réflexion, et d'un jeu de défamiliarisation entre les attentes et les effets de surprise. Ces éléments sont présents de façon plus ou moins prégnante dans chacune des scènes de poursuite de notre corpus et définissent leur esthétique sonore.

\section{ESTHÉTIQUE MUSICALE DE LA POURSUITE}

Les brusques variations de la cadence kinésique, une forte tension narrative, la prégnance des effets sonores (ou plutôt une hiérarchie sonore prônant la sensation), de même que la perméabilité, voire la dissolution des cloisons entre les éléments musicaux et les effets sonores sont à l'origine des caractéristiques principales de l'esthétique musicale des scènes de poursuite. Au même titre que la production de musique électronique, le recours aux outils informatiques est central dans le processus de création musicale du cinéma hollywoodien contemporain. En ce sens, l'approche compositionnelle de Hans Zimmer, et largement empruntée par les compositeurs associés à Remote Control,

relies on assembling ideas by use of music technology and hearing them played back by machines. Real orchestra parts may complement or be substituted for synthetic elements but they are added only later, at a stage when Zimmer's score is already conceptually advanced. [...] Zimmer himself points out that every note in every score of his has at some point been conceived, performed, and manipulated by him (Hexel 2016, p. 31-32).

L'emploi de médiums similaires entre l'Electronic Dance Music (EDM) et la création musicale au cinéma suppose, entre autres, des techniques de composition analogues, une corrélation entre leurs différentes approches pour le traitement du son enregistré, de même qu'une représentation visuelle de la composition musicale qui influence 
les techniques compositionnelles employées, notamment, par exemple, dans le cas du layering (voir ci-dessous). Plus précisément, la musique des scènes de poursuite partage avec l'EDM une mise en valeur de la dimension rythmique. Dans les deux cas, le rythme est conçu pour être davantage ressenti qu'écouté et sert de fondement à l'architecture sonore 9 . Certaines caractéristiques musicales admises pour 1'EDM se retrouvent d'ailleurs, outre le cadre de la poursuite, dans l'esthétique sonore globale de Remote Control : mesure à quatre temps, musique répétitive (cyclique), très fort volume, emphase des basses fréquences ( bass and sub-bass »), formées de la combinaison de diverses sources sonores (records, samples, etc.), importance accordée aux timbres (texture sonore), avec l'intervention de phrases musicales plus larges qui tendent à intervenir en multiples de mesures à quatre temps ${ }^{10}$. Trois pistes des albums qui ont servi à l'établissement de notre corpus forment des exemples pertinents pour illustrer les liens entre les caractéristiques musicales de l'EDM et la musique des scènes de poursuite (extraits audio 1, 2 et 3 ).

Extrait audio 1 : Mark Mothersbaugh, Thor. Ragnarok, "Sakaar Chase» (2017), 00:00-00:36. Écouter.

Extrait audio 2 : Harry Gregson-Williams, Total Recall, "Rooftop Chase» (2012a), 00:00-00:38. Écouter.

Extrait audio 3 : Lorne Balfe, Geostorm, "Lightning Chase» (2017), 02:51-03:42. Écouter.

Dans la foulée de Mark J. Butler, Mark Minett définit quatre aspects de base de l'EDM employés dans la création musicale filmique hollywoodienne contemporaine qui sont fondamentaux pour la définition de l'esthétique sonore de la poursuite, soit l'utilisation du sampling, la manipulation du rythme, le looping et le layering (Minett 2013, p. 195). Cette définition souligne l'importance du travail sur la texture sonore, sur les timbres et leur juxtaposition, et ce davantage qu'une réflexion musicale basée sur la logique mélodie-accompagnement. Chacun des aspects soulevés offre des réponses particulières aux fonctions auxquelles doit répondre la musique dans les scènes de poursuite.

\section{Échantillonnage (sampling)}

La vaste majorité des compositeurs associés à Remote Control procède par échantillonnage, ou sampling, pour la création musicale, c'est-à-dire :

Selecting, recording, editing, and processing sound pieces to be incorporated into a larger musical work [...] a creative musical endeavour that encompasses the selection, cultivation and presentation of aural fragments that can function in myriad recombinant, remixable forms (Rodgers 2003, p. 313 et 319).

L'ensemble des sons musicaux enregistrés sont ainsi appelés à être traités électroniquement. La synthèse sonore de même que le traitement synthétique des matériaux musicaux sont deux aspects notables de l'esthétique sonore de Remote Control, et qui

9 À ce propos, voir Butler 2006, p. 91.

10 Voir les caractéristiques énoncées par Butler (ibid., p. 8). 
marque une forte différence avec le néoclassicisme hollywoodien. À titre d'exemple, Hans Zimmer

tends to use numerous pick-ups and overdubs, such as instrumental effects that he may add to cues where needed. Because some recorded material may already exist prior to the scoring session (e.g., suites, demos, soloists) and because additional material may later be sourced from elsewhere, the orchestrated session score of a Zimmer cue sometimes looks rather bare on the page. The sound of recorded live elements will, furthermore, be edited and mixed, sometimes with striking effect. Zimmer fully exploits cutting-edge recording techniques (including the inventive use of different microphone positions) and music production tools (including analog and digital effects), to produce a more cohesively affective and impactful sound (Hexel 2016, p. 39).

Ce type d'utilisation de samples est notable dans la scène de poursuite en voiture de Total Recall (Len Wiseman, 2012), entre autres par l'emploi de notes isolées aux cuivres ou aux cordes pour structurer par le moyen de stingers différentes phases de l'action, ou encore pour renforcer la tension au cours des dialogues (voir l'analyse de la scène ci-dessous).

\section{Manipulation du rythme}

L'impulsion rythmique est, comme mentionné, l'élément musical le plus important de la scène de poursuite. Le jeu sur la perception temporelle à partir de la manipulation du rythme permet de susciter à la fois la tension et provoquer l'immersion, générer une perte de contrôle et un inconfort par l'opposition entre une vitesse excessive et des instants d'inertie qui structure, comme nous le verrons, le corps de la poursuite. À titre d'exemple, Minett souligne que le compositeur Graeme Revell emploie ce type de jeu d'opposition rythmique pour la musique du film Lara Croft. Tomb Raider (Simon West, 2001) afin de "mark off spectacle, to signal a pause in the action, and to create suspense " (Minett 2013, p.197). En cohérence avec le montage visuel, le type de composition que l'on retrouve dans les scènes de poursuite est basé sur des motifs répétés et davantage porté sur les jeux entre les timbres et les différents registres qui permettent de densifier l'ensemble sonore. De plus, un traitement musical qui se concentre sur le rythme et qui n'entend pas suivre de façon spécifique une des figures représentées à l'écran est à même de suggérer, comme dans les films d'horreur, une perte de contact avec le héros, une prise de distance avec ce dernier, ou encore figurer une situation périlleuse (Donnelly 2009, p. 116).

\section{Bouclage (looping)}

Au même titre que l'ostinato dont il constitue une forme moderne, le bouclage est basé sur la répétition périodique d'une cellule mélodique, rythmique, ou rythmico mélodique, via un traitement numérique. Cette technique compositionnelle est omniprésente dans les scènes de poursuite dont la structure musicale se fonde sur de brèves séquences répétitives et malléables, souvent à quatre temps. Le type de bouclage caractéristique des scènes de poursuite rejoint l'unité fondamentale de la structure musicale de l'EDM qui consiste en un 
repeating pattern associated with a particular instrument, which fans and musicians describe as "loop" [...] The primary indicator of loop-based structure is cyclical repetition [...] Generally, the term "loop " refers to a single instrumental pattern (or to a single repeating sample, as with looped breakbeats), though mutliple loops can be combined into repeating sequences (Butler 2006, p. 90).

La fonction de l'ostinato dans les scènes de poursuite, comme celle attribuée aux drones, est de susciter une augmentation sensible de la tension ${ }^{11}$. Toute réduction de différence entre des matériaux musicaux sert " to induce instant tension as well as having a cumulative effect of disquiet or extreme anxiety » (Donnelly 2010, p. 161) ${ }^{12}$. La scène de poursuite en voiture de Total Recall procède selon un tel principe de mise en tension par ostinato (voir analyse de la scène ci-dessous, notamment la deuxième partie du développement de la poursuite).

Stratification (layering)

L'intérêt porté aux timbres et aux registres instrumentaux (en solo ou par sections instrumentales) implique un abord de l'ensemble musicosonore par stratification (layering). Comme l'affirme Butler, " one might trace this textural individuality back to the recording process, which involves multichannel environments that allow many different textural layers to be controlled independently» (Butler 2006, p. 94). La composition musicale axée sur le jeu entre les timbres, les registres et la répétition de cellules rythmiques propres à chaque strate instrumentale rejoint ainsi l'EDM : "Instrumental sounds used in EDM "typically have distinctive timbres and registers, so that they are heard as individual layers rather than combining into groups". Thus, "texture in EDM consists of a number of quite distinct layers, relatively equal in prominence, that are constantly in flux" " (Minett 2013, p. 194). Les différentes strates de timbres, comme il sera démontré, peuvent être associées à différentes localisations (intérieures/extérieures, plans resserrés/plans d'ensemble, etc.), à différentes phases de la poursuite (densification des strates en lien avec la montée de la tension narrative), et aux entités opposées prenant part à la poursuite (dominance des timbres associés à un protagoniste en particulier). De telle manière, la stratification a une fonction musicale servant à structurer la narrativité par densification ou dissipation, ou par association à un protagoniste, soit une fonction semblable à celle que l'on attribue au leitmotiv en musique de film ${ }^{13}$. Enfin, cette approche compositionnelle permet une plus grande malléabilité dans le travail de la musique tout au long de la production filmique. À titre d'exemple, "in order to be able to adjust his music at every stage along the way, Zimmer uses numerous separate tracks in large Cubase or Pro Tools sessions. As a result, elements can

\footnotetext{
11 Dans le cadre de la musique des films d'horreur, Donnelly souligne "The currency of horror film music comprises a relatively small selection of musical devices [...] This includes the "stinger" or "sting", which manifests a physical shock in a musical blast, and the tension ostinato, a loop of music that provides tension through cumulative effect, along with its first cousin, the drone » (Donnelly 2005, p. 91). Ces éléments sont également fondamentaux dans les scènes de poursuite.

12 Voir aussi Lerner 2010, notamment la préface (p. iix-xi).

13 Sur la fonction leitmotivique dans la musique de film de l'âge d'or hollywoodien, voir notamment Wescott 1989.
} 
easily be added or withdrawn, instrumental choices revised, balances redressed " (Hexel 2016, p. 39). Cette dernière fonction, de même que l'importance accordée aux timbres dans la composition musicale, soulève la question de la perméabilité entre les cloisons de différents éléments de l'ensemble sonore.

\section{COMBINAISON DES DIVERSES SOURCES SONORES}

La prégnance des effets sonores dans les scènes de poursuite implique un emploi de la musique à des fins de mise en valeur de ceux-ci. Cette logique inhérente à l'esthétique musicale de la poursuite met en évidence le jeu sur le "fantastical gap » (Stilwell 2007) entre les sons de la diégèse et ceux non diégétiques, entre les effets sonores et la musique, et met à mal les limites entre la fiction et le réel, entre l'espace séparant la conscience et l'inconscience du film ${ }^{14}$. La musique de Mad Max. Fury Road (Miller, 2015) connaît une diégétisation particulièrement pertinente, de même qu'une dissolution des frontières entre l'espace diégétique et non diégétique (extrait vidéo 1).

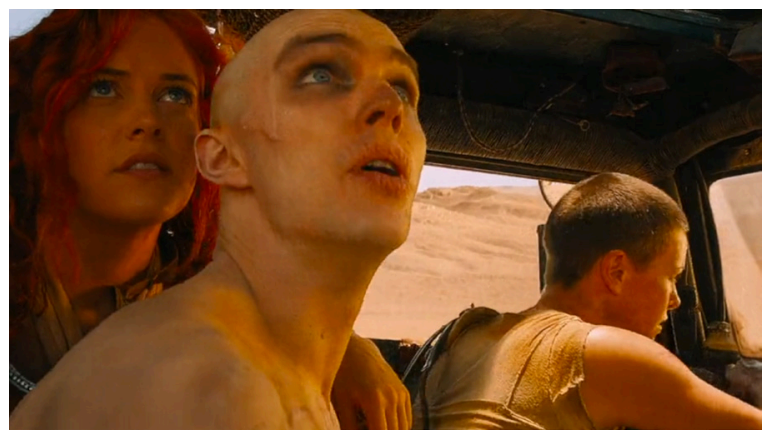

Extrait vidéo 1: George Miller, Mad Max. Fury Road, scène de poursuite finale (2015), 01:40:50-01:41:10 (C) Warner Home Video. La guitare électrique et les tambours innervent la partition musicale et sont justifiés par la diégèse.

Ce mélange entre les différentes sources sonores est renforcé par la primauté accordée aux timbres dans l'approche compositionnelle, de même qu'une redéfinition de la hiérarchie sonore traditionnelle. À titre d'exemple, lorsqu'une des entités de la poursuite est incarnée par un robot, sa signature sonique tend à intégrer la composition musicale, comme le son de l'essaim de drones dans Transformers. The Last Knight (Bay, 2017). Nous verrons de même dans l'analyse de la scène de poursuite en voiture de Total Recall, de quelle façon le recours aux sons synthétiques correspond à l'univers futuriste du film et peut y trouver une justification diégétique, à cheval entre l'effet sonore et la musique électronique.

\section{STRUCTURE FORMELLE DE LA POURSUITE}

De façon générale, les scènes de poursuite mettent en interaction deux entités narratives opposées qui sont singulières ou composées de divers éléments. Ces deux

14 À ce propos, Donnelly affirme : "There is a notable collapse of the space between diegetic sound and non-diegetic music. This manifests a collapse of mental space, between the film's "conscious" and its "unconscious" " (Donnelly 2009, p. 106). 
entités évoluent dans un rapport manichéen entre pourchasseur et pourchassé qui n'est toutefois pas déterminant de leurs identités narratives respectives. À titre d'exemple, on retrouve parfois une inversion des postures de force, comme dans les scènes de poursuite qui cadrent la structure narrative de Jason Bourne (Greengrass, 2016) où les scènes de poursuite initiale et finale sont symétriques, la proie devenant le prédateur et vice-versa. La proie de la poursuite n'est pas systématiquement incarnée par la figure du héros dans le contexte narratif filmique global ni le prédateur par les protagonistes rivaux. Bien que ce rapport dualiste entre la proie et le prédateur constitue le fondement de la logique narrative de la poursuite, d'autres éléments peuvent prendre part l'action, notamment pour figurer une entité plus neutre entre les pôles principaux. Cet élément supplémentaire est souvent incarné par les forces de l'ordre (l'armée, la CIA, le FBI, ou la police comme dans Ninja Turtles 2 (cue "Shredder Escape ») et Jason Bourne (cue "Strip Chase »). Aussi, tel que souligné par Arijon, la poursuite peut être double : "Le chasseur poursuit sa victime et tous deux sont poursuivis par un troisième intéressé par l'un d'eux. Si les deux premiers ignorent la présence du troisième, l'issue de la chasse dépend du comportement de ce dernier» (Arijon 2004, p. 485 ${ }^{15}$ ). La présence d'éléments supplémentaires au sein des entités principales (prédateur et proie) et secondaires est un moyen efficace pour dynamiser l'action de la scène. Par ailleurs, chacune des entités principales de la poursuite appelle un traitement musicosonore particulier qui permet de souligner leur position respective.

Réduite à sa plus simple expression, à l'exemple des films de poursuite du cinéma primitif, la scène de poursuite s'élabore selon une structure tripartite, soit une phase d'exposition, suivie d'un développement et d'une résolution ${ }^{16}$. La première phase permet une mise en place de la tension et un déclenchement de la poursuite, le développement fait l'objet d'une augmentation de la tension initiale, la plupart du temps par paliers, jusqu'à l'atteinte du climax et de la résolution finale.

\section{Prédateur}

Sur le plan narratif, la figure du prédateur est généralement impersonnelle, immorale, barbare, et souvent dissimulée sous le couvert d'un masque ou dans un véhicule (s'il n'est pas un véhicule ou un robot lui-même). Aussi, la structure de la meute est fréquente dans la représentation du prédateur (corps d'agents de la CIA dans Jason Bourne, la police dans Hitman's Bodyguard (Hughes, 2017) et Drive Hard (Trenchard-Smith, 2014), l'essaim de drones dans Transformers. The Last Knight, les

15 Nous ne pouvons toutefois abonder dans le sens d'Arijon concernant la dernière partie de son assertion, le troisième élément de la double poursuite n'étant pas nécessairement source du dénouement (par exemple, Ninja Turtles 2, cue «Shredder Escape » dans notre corpus).

16 «Dans Stop Thief, de l'anglais Williamson [...], la structure poursuite, extrêmement rudimentaire encore, se développe sur trois plans seulement, chiffre dont on verra qu'il faut tenir pour un minimum absolu afin que la poursuite puisse devenir une forme narrative complète. [...] Chez Williamson, le premier plan (exposition) montre le ressort de la poursuite [...] ainsi que le départ ; le second (développement) en montre le cours [...] ; le troisième y met un terme (résolution)» (Burch 1983, p. 34). Sur la poursuite dans le cinéma des premiers temps, voir aussi Auerbach 2000 et McCaffrey 2016. 
motocyclistes dans Ninja Turtles 2), de laquelle se dégage un protagoniste particulier (Lori Quaid (Kate Beckinsale) dans Total Recall, la guerrière Sakaraan en jaune dans Thor (Waititi, 2017), Asset (Vincent Cassel) dans Jason Bourne).

L'inhumanité du prédateur et la dynamique de la meute marquent son identité sonique. Il est ainsi généralement représenté par des effets sonores (coups de feu, alarmes et autres sirènes, divers sons de véhicules comme les drones dans Transformers. The Last Knight), ou par interjections qui, souvent, sont altérées de façon synthétique. À ce titre, même lorsqu'il s'agit d'une voix de source organique (ou respiration), elle est soit altérée par un filtre de synthèse (comme dans Thor. Ragnarok ou Mad Max), soit quasi muette (Jason Bourne, Geostorm (Devlin, 2017), Java Heat (Allyn, 2013)). L'incarnation synthétique de la figure sonore du prédateur lui confère un aspect surnaturel, dominant la proie qui est de nature plus organique. Par ailleurs, l'identité sonique du prédateur tend à inonder l'ensemble sonore diégétique de la poursuite, renforçant ainsi son impression de puissance, d'envahissement et de domination, à l'exemple de motocyclistes dans Ninja Turtles 2 ou de la meute sous l'égide de Immortan Joe dans Mad Max (extraits vidéo 2 et 3 ).

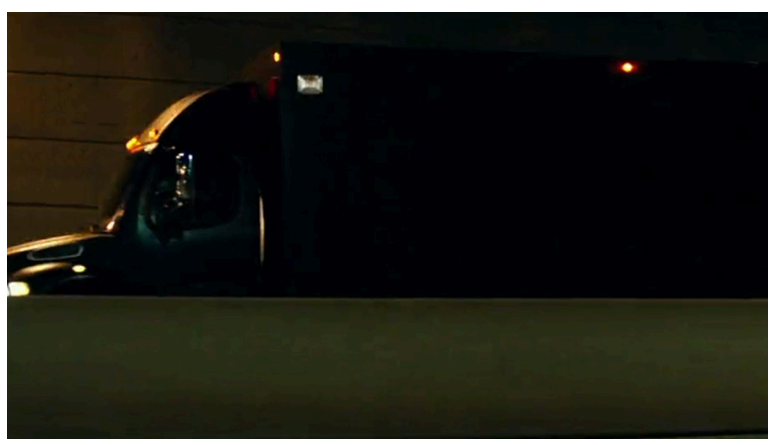

Extrait vidéo 2 : David Green, Teenage Mutant Ninja Turtles. Out of the Shadows, poursuite entre les motocyclistes de Shredder et les forces de l'ordre (2010), 00:16:41-00:17:40 (C) Paramount Pictures. Le son des motocyclistes dominent l'environnement sonore.

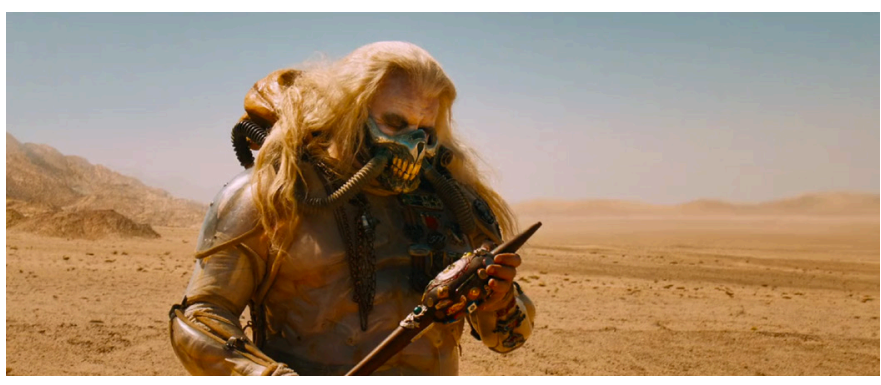

Extrait vidéo 3 : George Miller, Mad Max. Fury Road, présentation du groupe sous l'égide d'Immortan Joe au début de la poursuite finale (2015), 01:29:06-01:30:01 (C) Warner Home Video. Les sons des véhicules, des tambours et de la guitare électrique dominent l'environnement sonore.

Proie

Contrairement au prédateur, la proie est figurée par une présence accrue de sources sonores organiques. Elle présente davantage d'interactions vocales (interjections et dialogues), de souffles, de cris, ainsi que différents sons qui marquent l'effort physique, 
témoins d'une certaine vulnérabilité. Alors que le prédateur tend à envahir l'ensemble sonore, la proie est présentée de façon plus intimiste (extrait vidéo 4).

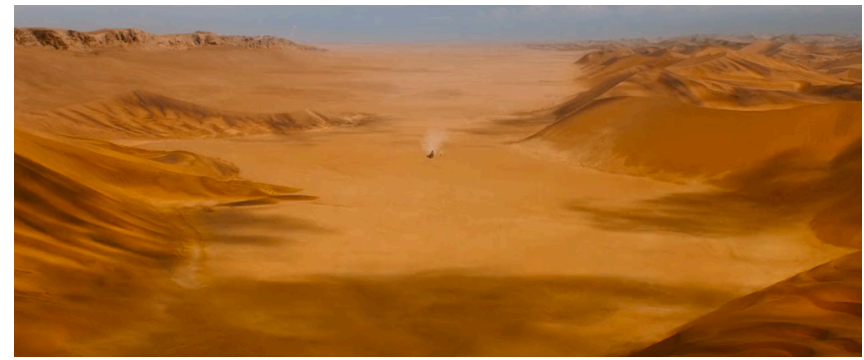

Extrait vidéo 4: George Miller, Mad Max. Fury Road, présentation de la proie de la poursuite finale (2015), 01:30:10-01:30:31 @ Warner Home Video. Le son de la boîte à musique et les murmures de la prière marquent l'identité sonore de la proie.

Toutefois, on retrouve à l'occasion une figuration extradiégétique de la proie par des thèmes musicaux lorsqu'elle est incarnée par le héros. Ces thèmes se dégagent de l'ensemble sonore à des moments qui ponctuent une reprise de force, et relancent la dynamique de la poursuite (extraits vidéo 5 et 6).

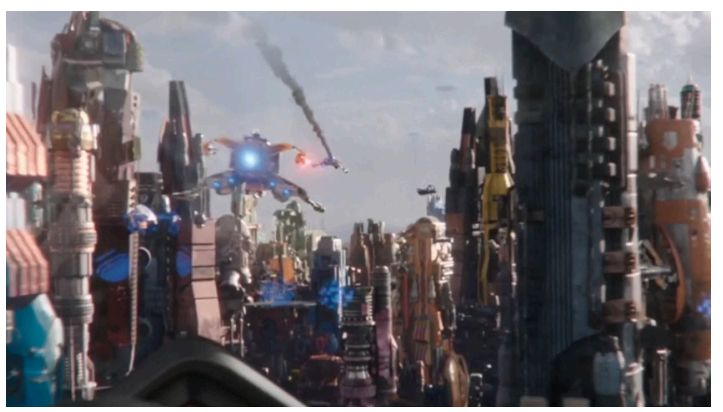

Extrait vidéo 5 : Taika Waititi, Thor. Ragnarok, fuite de Thor (Chris Hemworth),

Bruce Banner (Mark Ruffalo) et Valkyrie (Tessa Thompson) de Sakaar (2017), 01:33:23-

01:33:58 @ Walt Disney Studios. Thème du héros évoqué alors que Valkyrie, suite à la destruction de son vaisseau, saute sur le vaisseau ennemi pour reprendre le combat.

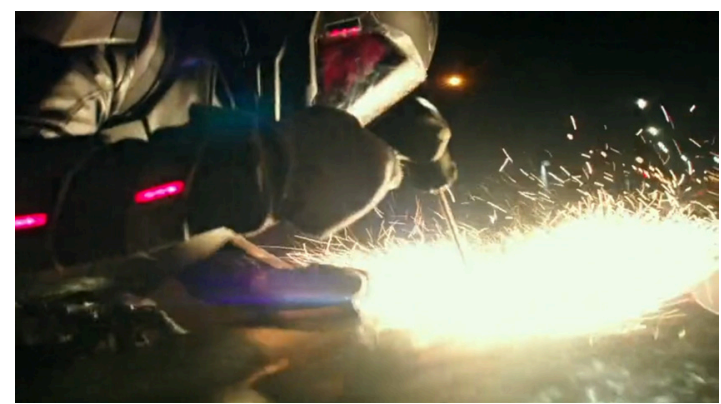

Extrait vidéo 6: David Green, Teenage Mutant Ninja Turtles. Out of the Shadows, thème des tortues ninja soulignant leur arrivée dans la poursuite pour capturer Shredder (Brian Tee) (2016), 00:18:45-00:18:58 C Paramount Pictures.

Prélude à la poursuite : exposition

L'articulation de la phase initiale de la poursuite est plus difficile à déterminer que celles des phases subséquentes. L'exposition fait l'objet soit d'une instauration 
généralement lente de la tension, ou alors prend place au sein d'un affrontement auquel succède la fuite d'un des belligérants (par exemple dans Total Recall (cue « Rooftop Chase "), Java Heat ou Geostorm). Elle est presque systématiquement justifiée par la narration et prévisible. Cette phase initiale permet de présenter les entités principales qui participeront à la poursuite (figures et véhicules), parfois l'environnement où elle se déroulera (parcours), et son événement déclencheur (embrayeur narratif). On y favorise dans la plupart des cas l'économie sonore afin de marquer de façon nette l'impact du déclenchement de la poursuite. Il s'agit également d'une phase importante au point de vue de l'engagement narratif pour susciter l'empathie envers les héros prenant part à la poursuite.

\section{Cartographie}

L'engagement spatial, basé sur la représentation mentale d'un espace géographique, constitue, tel que mentionné, l'un des cadres du modèle d'engagement dans les jeux numériques proposé par Calleja (Calleja 2007, p. 83). Calleja affirme que " the knowledge of an area creates a sense of habitation and belonging to the region. This internalization process is crucial for the achievement of a sense of comfort and place, both in the physical and digital worlds " (ibid., p. 88). Une disposition similaire est notable dans les scènes de poursuite au cinéma, notamment pendant la phase d'exposition, mais aussi dans le développement de la poursuite. De même, une alternance entre la présentation des entités de la poursuite et des points de vue généraux est un moyen efficace pour dynamiser la scène et créer la tension via la perte de repères visuels des protagonistes. Parmi les types de cartographies récurrentes, on en retrouve une que l'on pourrait qualifier " d'intention », ayant pour fonction narrative d'illustrer l'objectif à atteindre, qu'il s'agisse d'un objet, d'un lieu, d'une personne, etc.

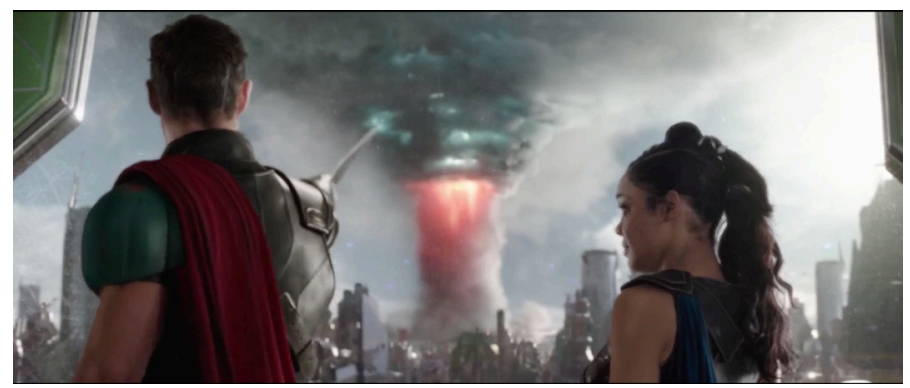

Figure 1 : Cartographie, Taika Waititi, Thor. Ragnarok (2017), 01:25:38 (C) Walt Disney Studios. Thor indique à Valkyrie l'issue par laquelle ils pourront fuir la planète Sakaar, avant le début de la scène de poursuite.

Ensuite, une deuxième forme, plus fréquente, peut être qualifiée de cartographie " globale », ou " d'ensemble ", où le plan visuel présente une vue aérienne de l'environnement où a lieu la poursuite. Ce type de cartographie est souvent justifié diégétiquement, notamment par la présence d'un hélicoptère dans Drive Hard, Total Recall, Ninja Turtles 2, et Green Zone (Greengrass, 2010). 

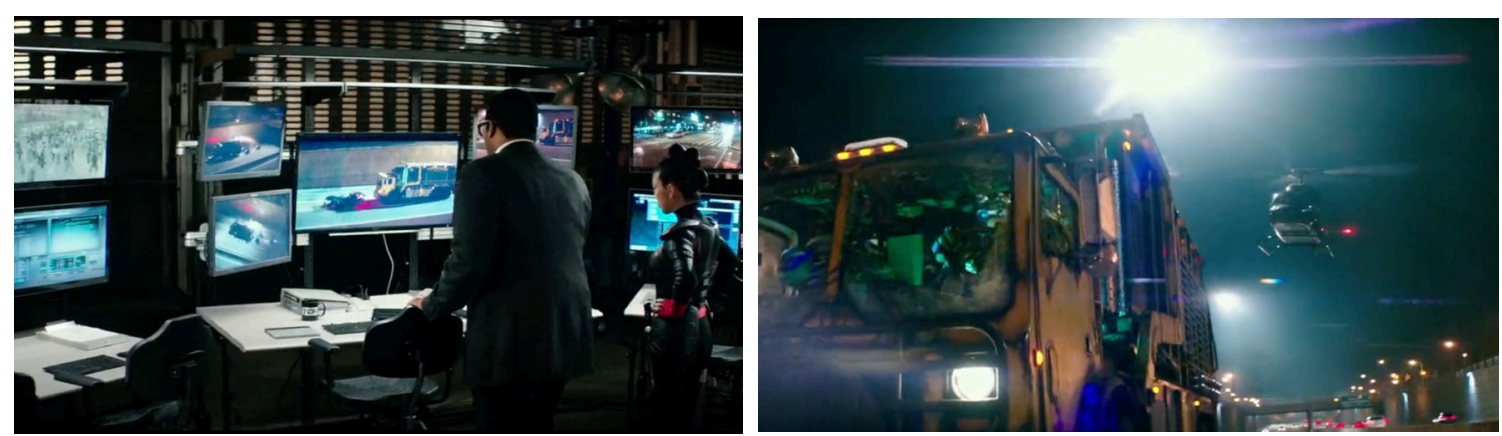

Figures 2 et 3 : Cartographies, David Green, Teenage Mutant Ninja Turtles. Out of the Shadows (2016), 00:20:52 et 00:20:54 @ Paramount Pictures.

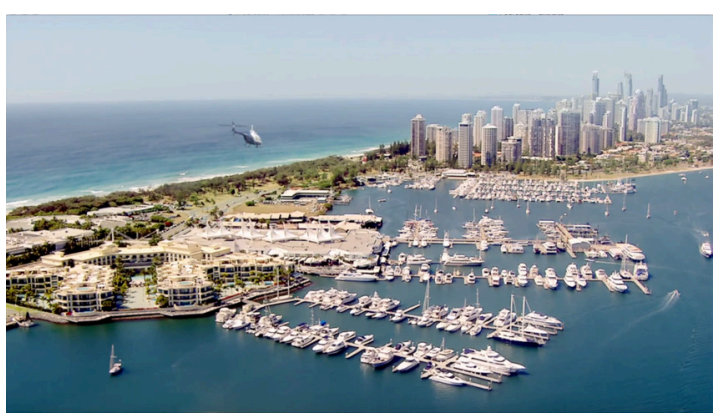

Figure 4: Cartographie, Brian Trenchard-Smith, Drive Hard (2014), 00:15:10 (c) Image Entertainment.

Enfin, une autre forme fréquente de cartographie peut être qualifiée de " numérique ». Cette dernière présente une esthétique visuelle proche des jeux vidéo avec une figuration ou simulation écranique des différentes entités prenant part à la poursuite.

Figure 5 : Cartographie, Paul Greengrass, Jason Bourne (2016), 00:28:15

(C) Universal Pictures.
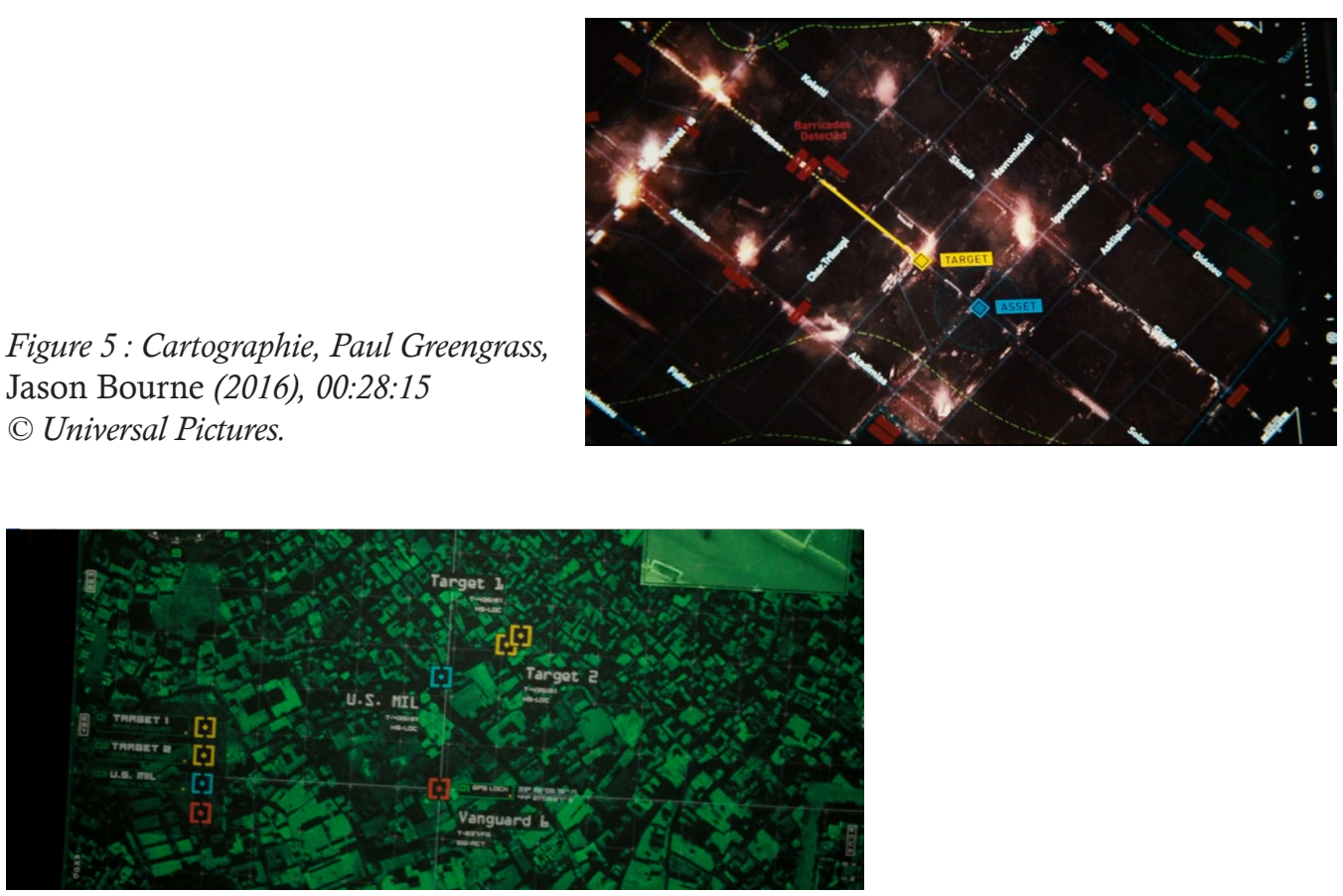

Figure 6: Cartographie, Paul Greengrass, Green Zone (2010), 01:31:45 @ Universal Pictures. 
$\mathrm{Au}$ niveau sonore, le recours à la cartographie permet de justifier diégétiquement l'environnement et le continuum sonores qui sont largement plus denses que le cadre du champ visuel ${ }^{17}$. Le plan cartographique constitue ainsi une forme de diégétisation sonore non déterminée. Ce procédé permet de suggérer rapidement le champ des possibles sources diégétiques, d'affirmer le réalisme de l'ensemble sonore, et d'encourager l'immersion spectatorielle via le renforcement du réalisme ${ }^{18}$. Enfin, en lien avec les propos d'Arijon, la cartographie se présente comme une manière de mettre en valeur une action visuelle :

Dans une bataille de grande envergure, les scènes de foule où l'action vagabonde doivent être alternées avec des scènes d'action où le ou les héros sont engagés dans un combat personnel. C'est un schéma de dilation - contraction pour filmer une action qui incite le spectateur à se déplacer continuellement du général au particulier et inversement. De cette manière, les personnages principaux sont intégrés au spectacle et leur relation à l'ensemble peut être ressentie par une identification plus forte aux personnages. Ces scènes de foule ainsi que celles où les protagonistes sont isolés, doivent avoir un rapport avec l'action centrale (Arijon 2004, p. 494).

\section{Déclenchement de la poursuite et embrayeur narratif}

Le déclenchement de la poursuite est généralement marqué par un effet de synchrèse ${ }^{19}$ entre un événement visuel et une interjection ou un effet sonore qui est déterminant pour la structure musicale. De plus, l'effet de synchrèse au début de la poursuite fait souvent l'objet d'un stinger correspondant à un choc ou une révélation. Ce type d'effet est d'ailleurs souligné comme agent immersif selon une étude menée par Russell Shilling, Michael Zyda et E. Casey Wardynski sur un jeu "First Person Shooter " : "The authors [...] observed that immersion is crucially enhanced by precise synchronization of sound and action displayed on the screen; this is objective (but not conclusive) evidence, in the context of digital games, for the effect of synchresis » (Shilling 2002 ${ }^{20}$ ). Dans le même ordre d'idées, Donnelly met de l'avant la participation de la musique pour stimuler la tension nerveuse, et l'importance du stinger à cet effet : "The most primitive (and most primal) musical moments in cinema are stingers, which engage with the audience on the most basic levels " (Donnelly 2005, p. 95) (extraits vidéo 7 et 8 ).

17 Cette fonction rappelle celle du son de situation (« establishing sound») employé au début des années 1930. Voir à ce propos Altman 2013.

18 Sur l'importance du réalisme pour l'immersion dans les jeux numériques, voir McMahan 2003. De même, les codes du réalisme sont l'un des trois thèmes généraux soulignés dans le survol de théories de l'immersion de Grimshaw (Grimshaw 2012, p. 358).

19 Michel Chion donne la définition suivante de la synchrèse : "Nom forgé que nous donnons à un phénomène psycho-physiologique spontané et réflexe, universel, dépendant de nos connexions nerveuses, et ne répondant à aucun conditionnement culturel, et qui consiste à percevoir comme un seul et même phénomène se manifestant à la fois visuellement et acoustiquement la concomitance d'un événement sonore ponctuel et d'un événement visuel ponctuel, dès l'instant où ceux-ci se produisent simultanément, et à cette seule condition nécessaire et suffisante » (Chion 1990, p. 55-57).

20 Cet article est cité par Grimshaw 2012, p. 361. 


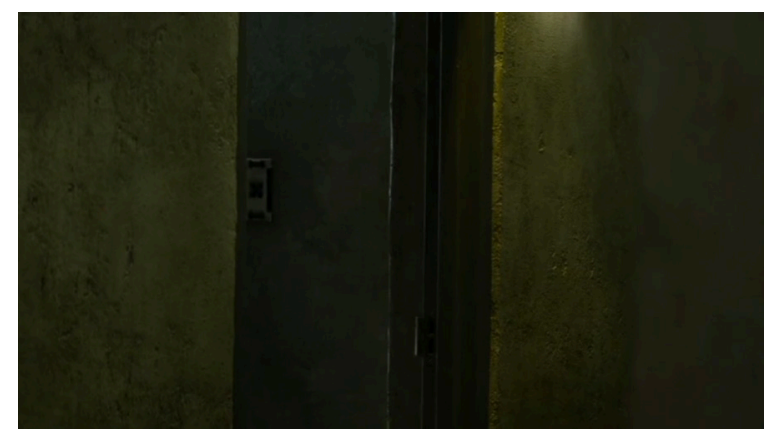

Extrait vidéo 7 : Len Wiseman, Total Recall, début de la poursuite entre Douglas Quaid (Colin Farrell) et Lori Quaid (Kate Beckinsale) (2012), 00:37:01-00:37:18 — Sony Pictures. Des sons synthétiques accompagnent le saut de Lori, marqué ensuite par un plan des deux protagonistes et le début de la poursuite sur un motif aux percussions.

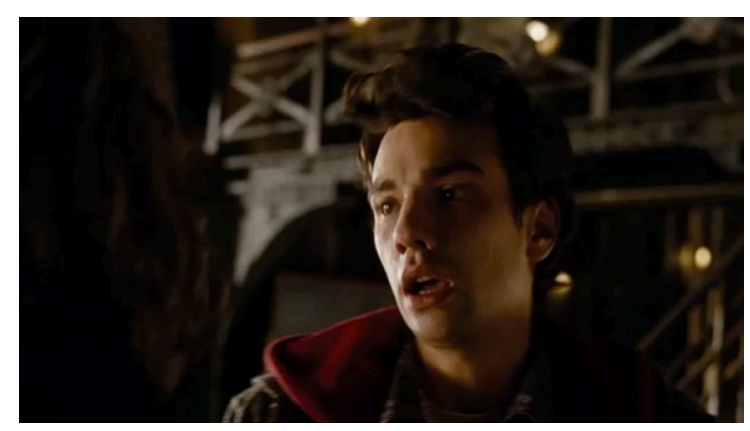

Extrait vidéo 8: Jon Turtletaub, Sorcerer's Apprentice, début de la poursuite entre Balthazar (Nicolas Cage) - Dave (Jay Baruchel), et Horvath (Alfred Molina) - Drake Stone (Toby Kebbell) (2010), 01:30:10-01:30:31 C Walt Disney Studios.

Départ de Horvath et Drake (au volant) marqué par un plan sur le crissement de la roue, le début du motif aux percussions et l'ostinato aux cordes.

\section{Développement de la poursuite}

La partie centrale, ou corps de la poursuite, est d'une durée aléatoire et structurée en différents paliers qui articulent la croissance de la tension. Cette phase est celle qui permet, grâce à sa logique structurelle, une expansion de la durée de la scène. $\mathrm{Au}$ même titre que les scènes de combat ou les musical numbers, comme l'indique David Bordwell (Bordwell 2006, p. 104), la scène de poursuite peut être étendue de façon indéfinie. De fait, cette phase centrale est sujette à des rebondissements qui vont relancer l'action. Elle est malléable et flexible, à l'instar des éléments musicaux qui y participent. Le contenu narratif du développement est relativement mince, favorisant l'effet spectaculaire. Si sa structure s'organise effectivement en paliers, chacun d'eux fait l'objet d'une montée en tension vers un climax dans l'aigu et une densification de l'ensemble sonore, avant une suspension de l'ensemble ou de la quasi-totalité des éléments sonores. La montée en tension est produite, entre autres, par la densification des strates sonores (layering), qui vont élargir le registre et la texture sonore, l'accélération de la cadence rythmique (montage plus serré et rapide), et les effets de dissonance. 


\section{Suspension temporelle}

Les scènes de poursuite sont dynamisées par une alternance entre une vitesse effrénée et des séquences de suspension temporelle (ou ralenti drastique) de la cadence rythmique qui constituent une forme d'agent immersif particulièrement efficient, jouant un rôle fondamental dans l'engagement avec la temporalité filmique. Selon Barker, "chase films that use slow-motion cinematography in this way play upon our fears of inertia and powerlessness by making us want to run and holding us back at the same time " (Barker 2009, p. 118). Ce sentiment d'impuissance est marqué au niveau visuel par un statisme de la caméra, un effet de limitation dans le déplacement au sein du plan qui expose nos limites corporelles suscitant un effet déstabilisant, tout comme les limites des cadres et du temps d'observation. Par ailleurs, la mise en relief des moments périlleux vécus par le héros au moyen de la suspension temporelle permet d'affermir l'engagement narratif du spectateur ${ }^{21}$. Un exemple phare de suspension temporelle dans notre corpus survient à la fin de la scène de poursuite en voiture de Total Recall (voir analyse de la scène ci-dessous).

La suspension temporelle a généralement lieu suite à une montée en tension de l'ensemble des éléments sonores, et se présente soit de façon totale (comme dans Total Recall), par de longues notes tenues apparentées aux drones (suspension des percussions et de la cadence rythmique), ou par une transition marquée dans la texture sonore (modification des timbres, par exemple des soli de cordes en notes tenues, ou mise en évidence d'une source sonore comme dans le cas de l'accident d'hélicoptère de Green Zone) ${ }^{22}$ (extrait vidéo 9).

Extrait vidéo 9: Paul Greengrass, Green Zone, bombardement de l'hélicoptère des forces de l'ordre américaines (2010), 01:33:59-01:34:46 @ Universal Pictures. Il y a une montée en tension de l'ensemble orchestral avant sa suspension, l'intervention de l'alerte sonore signifiant l'imminence de l'impact, puis l'explosion, sa décomposition en plusieurs plans visuels, de même que divers points d'écoute, sans occurrences musicales ou vocales.

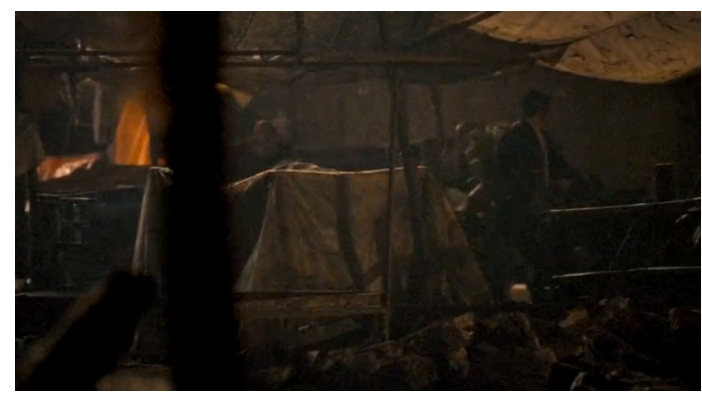

Ces moments de suspension, fortement employés dans les scènes d'action et dans la musique des films d'horreur, sont comparés à la musique pop ou l'EDM par Donnelly comme des moments de «drop-out» qui offrent également des instants d'anticipation,

21 Arijon souligne en ce sens : «Des scènes de mort ont été filmées au ralenti sur l'écran. Akira Kurosawa, avec ses Sept Samuraï a inauguré cette pratique. Quelques effets intéressants et surprenants sont à noter, en particulier, dans The Wild Bunch de Sam Peckinpah, qui, en plein milieu d'une scène frénétique, introduit des morts en mouvement ralenti »(Arijon 2004, p. 501).

22 Ce procédé rejoint ainsi celui de la retenue de la pulsation rythmique dans l'EDM : «As a phenomenon, withholding the beat is both textural and metrical» (Butler 2006, p. 92). 
de préparation au retour de la musique et du son avec plus d'intensité 23 . De fait, on peut associer la suspension temporelle à la suppression du bass drum pendant une performance EDM, ou au procédé de "withholding the beat » ou "breakdown ». Cette pratique est courante pour susciter une attente frénétique. Comme le souligne Minett :

[Withholding the beat] is a common practice used to build anticipation within the EDM audience; as dancers anticipate the return of the beat, their energy often builds to a frenzy.

"Dropping the beat", or returning the bass drum after its absence, is a technique used to climactic effect (Minett 2013, p. 195).

La suspension temporelle joue de même sur une transition rapide entre des pôles extrêmes, entre une grande densité sonore et le silence, entre un rythme excessif et sa suspension. Enfin, un effet de synchrèse marque souvent la reprise ou la fin de la poursuite (extrait vidéo 10 ).

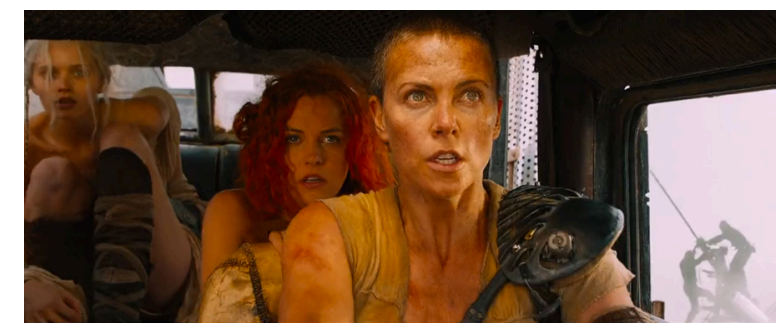

Extrait vidéo 10 : George Miller, Mad Max. Fury Road, situation critique pour Max Rockatansky (Tom Hardy) et Imperator Furiosa (Charlize Theron) pendant la scène de poursuite finale (2015), 01:37:12-01:38:13 @ Warner

Home Video. On note une suspension de l'ostinato des cordes dans l'aigu et de l'environnement sonore de la poursuite alors que Max chute du véhicule, rattrapé de justesse par Furiosa. Ensuite, la reprise des cordes en ostinato marque un retour de la tension jusqu'au poignardement de Furiosa, suivi d'un autre type de suspension temporelle, cette fois basé sur une modification de l'ensemble sonore, présentant un long motif aux cordes et aux cuivres.

Résolution

La résolution met un terme à la croissance de la tension (forte intensité, accélération du rythme, montée vers l'aigu, des dissonances de plus en plus accentuées, etc.) soit en instaurant un ensemble musicosonore en contraste avec celui mis en place lors du développement, ou par l'intervention d'un élément non musical structurant qui vient marquer la conclusion de la scène. Cette phase peut être l'objet d'une suspension temporelle permettant de créer un effet de relâchement dela tension, parfois via des notes tenues (Transformers. The Last Knight), par un changement dans l'effectif instrumental (The Hitman's Bodyguard), ou encore par le silence (Jason Bourne, Ninja Turtles 2, cue "Shredder Escape ", Total Recall, cue "Car Chase " et cue "Elevator Chase »). La résolution de la tension peut également subvenir via un effet de synchrèse fortement marqué, notamment lorsque l'issue d'une poursuite figure un accident (Run All Night (Collet-Serra, 2015), Total Recall (cue « Car Chase»), Geostorm). Enfin, certains cas ne présentent pas des propriétés aussi conclusives et forment une transition musicale vers 
la scène suivante (comme dans Java Heat) ou marquent une dissolution des éléments sonores mis en place pendant la poursuite (comme dans Total Recall (cue « Rooftop Chase »). Ces approches, qui agissent par dissipation plutôt que par résolution de la tension, témoignent d'une ouverture narrative de la poursuite et de l'aspect non définitif de son dénouement (extrait vidéo 11).

Extrait vidéo 11 : Conor Allyn, Java Heat, capture de Jake Wilde (Kellan Lutz) par les forces de l'ordre et fin de la poursuite (2013), 00:37:01-00:37:18 (C) IM Global. Le fondu enchaîné sur la mosquée est accompagné d'un fondu similaire de la partie musicale, non conclusive.

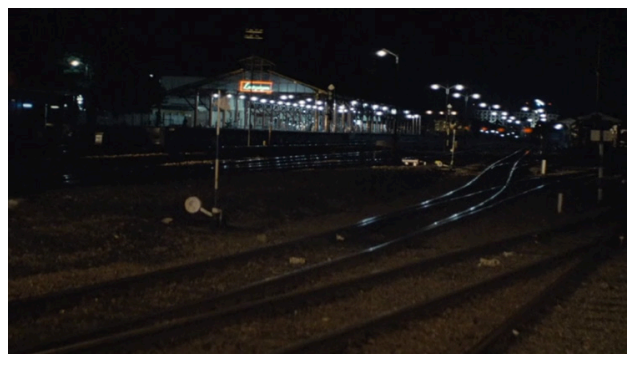

AnAlyse De LA SÉQuence de POURSuite EN VOITURE DANS Total RECALL

Afin de résumer et d'observer l'application des réflexions émises au cours de la première partie de notre article, cette section entend proposer une analyse plus élaborée d'une séquence de poursuite dans son ensemble, soit celle de la poursuite en voiture dans Total Recall (Len Wiseman, 2012, musique de Harry Gregson-Williams). Total Recall est le remake du film éponyme de Paul Verhoeven (1990, musique de Jerry Goldsmith), adapté de la nouvelle de Philip K. Dick, We Can Remember It For You Wholesale (1966). En tant que film d'action et de science-fiction, l'adaptation de Wiseman présente des liens étroits avec des productions anthologiques du genre comme Blade Runner (Ridley Scott, 1982), The Fifth Element (Luc Besson, 1997), Minority Report (Steven Spielberg, 2002), et, avec nombre de citations, le film original de Verhoeven. L'intrigue prend place au sein d'un futur post-apocalyptique à la fin du $\mathrm{XXI}^{\mathrm{e}}$ siècle où la terre, largement inhabitable à la suite des conflits chimiques, est divisée en deux territoires, l'Union fédérale britannique (UFB) et la Colonie (ancienne Australie). Plusieurs résidents de la Colonie vont travailler aux usines de l'ufB via « la chute ", un ascenseur de gravité voyageant à travers le centre de la Terre. Parmi ceux-ci, Douglas Quaid (Colin Farrell) est un ouvrier dont la vie morne et la hantise de rêves récurrents poussent à visiter Rekall, une société d'implantation de mémoires artificielles. Alors qu'il y découvre sa potentielle identité d'ancien agent secret s'entame une dynamique de fuite perpétuelle face aux forces de la police de l'uFB et à sa femme, Lori Quaid (Kate Beckinsale), agente secrète au service de l'UfB. Au cours de sa fuite, Douglas fait la rencontre de Melina (Jessica Biel), perçue en rêve, qui collabore avec les forces rebelles de la Résistance. Le fil rouge de la narration tisse un doute constant par rapport à la réalité des perceptions du héros et son identité.

Le montage visuel frénétique associé à une haute résolution de l'image (48 images/ seconde), une caméra mobile (longs travellings, séquences de caméra à l'épaule, panoramiques en circonvolution), et une décomposition des plans rapprochés sur les protagonistes, génère une impression de mouvement constant tout au long du film. Du point de vue musical, Total Recall constitue une illustration prégnante de 
l'esthétique des films d'action hollywoodiens des années 2010 et de la signature sonore de Remote Control. De telle manière, la fonction principale dont procède la musique est d'appuyer l'atmosphère générale du film en répondant aux besoins narratifs du scénario de poursuite où se perpétue inlassablement la menace létale des forces de l'ordre contre le héros. La partition résultante de la collaboration entre Gregson-Williams et le groupe de musique électronique Hybrid irrigue l'ensemble du film. Elle agit comme élément dynamique qui soutient l'action et met en valeur les effets sonores, accordant une emphase à l'intensité du flux rythmique qui traverse le métrage. La trame musicale du film se fonde ainsi sur un amoncellement de rythmes, de nappes, de drones et d'ostinati jonchés d'interventions mélodiques et de sons synthétiques. La partie orchestrale, outre quelques motifs pianistiques et l'importante batterie de percussions, se concentre autour de l'emploi de cuivres (notamment pour des stingers ou des motifs mélodiques simples) et de cordes (principalement sous forme de nappes ou d'ostinati). De plus, les instruments acoustiques sont abordés selon leurs qualités timbrales pour générer des effets d'atmosphère, comme le motif récurrent de descente dans l'aigu aux cordes qui simule la chute, ou encore les échanges d'accords dissonants aux cuivres dans des registres opposés qui induit une tension.

La scène de poursuite en voiture qui nous occupe intervient alors que Douglas, identifié à la suite du contrôle douanier de la chute, est secouru par Melina au cours de sa fuite.

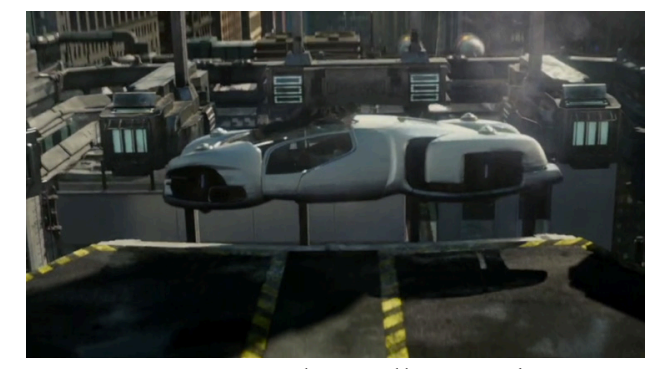

Extrait vidéo 12 : Len Wiseman, Total Recall, scène de poursuite en voiture (2012), 00:52:03-00:55:07 (C) Sony Pictures.

\section{Prédateur et proie}

La figure du prédateur correspond à la structure de la meute, composée d'un groupe de soldats-robots de la police de l'UFB dirigé par Lori Quaid. L'apparence des soldats évoque à la fois celle des Stormtroopers de la saga Star Wars et les humanoïdes de I, Robot (Alex Proyas, 2004), affichant un aspect noir et blanc, le visage couvert et impersonnel. Lori porte, depuis le dévoilement de son identité d'agente secrète au début du film, les cheveux détachés qui couvrent une partie de son visage. L'identité sonique de la meute est déterminée par le son des sirènes de police qui envahissent l'espace sonore. De plus, la première intervention vocale du prédateur (ordre vocal de la centrale) est filtrée synthétiquement par radio, alors que Lori demeure muette pendant toute la première partie de la poursuite. La figure du prédateur est constamment présente au cours de la séquence. Elle envahit l'espace diégétique et celui de la proie, et ce même lorsque les véhicules de la police de l'UfB ne sont pas visibles dans le champ, soit par le son des sirènes, soit par le reflet des lumières des sirènes sur le véhicule de la proie. 
Cette dernière est incarnée par le véhicule de Melina et Douglas. Les deux protagonistes échangent plusieurs dialogues au cours de la première partie de la poursuite, semblent constamment à bout de souffle, et sont présentés comme étant confinés dans l'espace du véhicule.

\section{Exposition}

L'avènement de la poursuite en voiture est prévisible et justifié par la narration. Les entités narratives de la proie et du prédateur sont toutes deux campées, de même que les véhicules prenant part à la poursuite et la géographie de son action. Cette phase d'exposition, sans occurrences musicales, présente également l'environnement sonore servant de trame de fond à la poursuite, soit celui de la route aérienne de l'UFB. En accord avec la division des pistes de l'album du film (Gregson-Williams 2012b et 2012 c), la séquence de poursuite s'articule en deux grandes parties, divisées par un déplacement du lieu de l'action vers un étage inférieur et inversé de la route aérienne. L'élément déclencheur de la poursuite, qui joue un rôle structurant pour marquer le début de la trame musicale, est déterminé par l'arrivée de la police - figurée par le son des sirènes au moment de l'ouverture de la porte du véhicule de la proie - et l'ordre de Melina adressé à Douglas : "Get in! Now! ». Cette interjection est soutenue par un crescendo en dissonance aux cuivres $(m i-f a)$, suivi d'un premier motif aux percussions et de stingers aux cuivres (d'abord sur sib avec la fermeture de la porte du véhicule de la proie, puis sur sol grave qui marque le début du développement de la poursuite et la tonalité de sol mineur ${ }^{24}$ ).

\section{Développement - Première Partie}

La partie initiale du développement de la poursuite se subdivise en deux paliers. Le premier présente un jeu d'alternance entre des plans d'ensemble de la poursuite et des plans resserrés à l'intérieur du véhicule de la proie. Ces derniers donnent lieu à des dialogues entre Melina et Douglas qui résument les liens entre les différents protagonistes. Les dialogues sont accompagnés de nappes aux cordes dans l'aigu illustrant la tension, la vulnérabilité et la situation critique dans laquelle se trouve la proie, le tout évoluant sur un rythme continu aux percussions qui soutient la dynamique de la poursuite. L'entrée de la première nappe de cordes correspond à l'interrogation de Douglas : "Do you know who I am? ", appuyé par un ré grave aux cuivres marquant la fin du motif descendant aux cuivres sol-fa-ré25.

24 Dans la version filmique, le sol grave aux cuivres n'est pas suivi du premier ostinato aux cordes, ni des trilles subséquentes, alors que ces éléments dominent la piste sonore correspondante de l'album (Gregson-Williams 2012b).

25 Le sol intervient au début de la poursuite, puis le fa accompagne l'affirmation de Melina : "I can't believe. It's you ". 


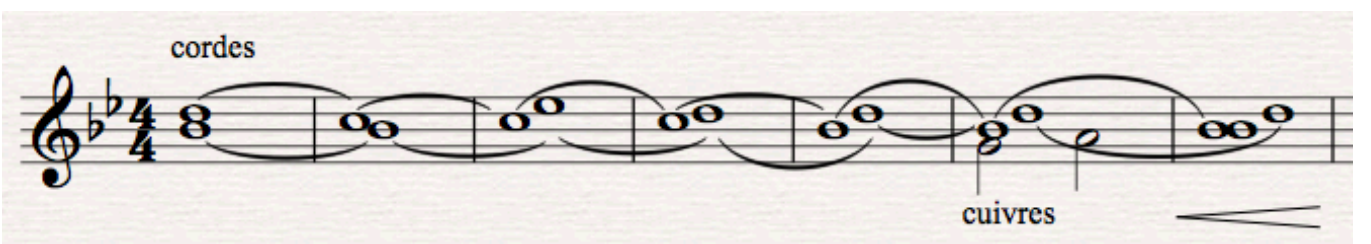

Figure 7: Harry Gregson-Williams, Total Recall, scène de poursuite en voiture, premier motif de nappes de cordes dans l'aigu et motif ascendant aux cuivres. Transcription personnelle à partir du film (00:52:49-00:53:00).

L'intensification des sirènes de police annonce l'arrivée du prédateur au niveau de la proie, accentuée par l'entrée d'un motif ascendant aux cuivres en crescendo (deux dernières mesures de la figure 7). Un stinger structurant sur un sol grave aux cuivres souligne le premier plan d'ensemble qui réunit les deux entités de la poursuite. Le retour au plan intérieur du véhicule de la proie est de nouveau marqué par un sol grave aux cuivres avec des nappes de cordes dans l'aigu, toujours soutenues par les percussions, alors que l'environnement sonore de la route aérienne se fait moindre. Outre une meilleure intelligibilité des dialogues entre Melina et Douglas, le recours aux nappes de cordes dans l'aigu soutient d'une part la tension, et modère d'autre part la dynamique de l'action au cours de ces mises au point narratives.

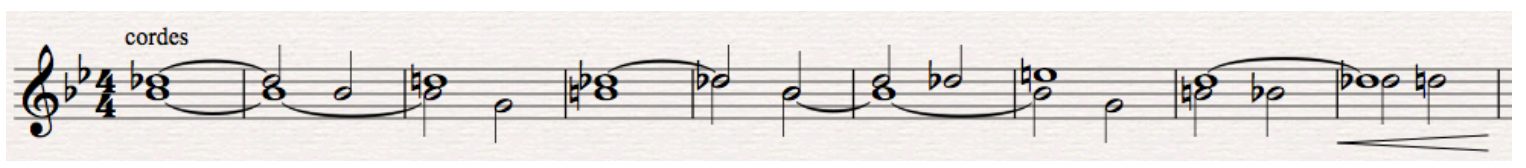

Figure 8: Harry Gregson-Williams, Total Recall, scène de poursuite en voiture, deuxième motif de nappes de cordes dans l'aigu. Transcription personnelle à partir du film (00:53:06-00:53:17).

De nouveau, un stinger sur un sol grave aux cuivres souligne le retour au plan d'ensemble de la poursuite, cette fois juxtaposé avec un lab en dissonance dans l'aigu aux cuivres et une intensification du son des sirènes de police. Les dissonances entre les registres opposés aux cuivres font place aux motifs descendants des cordes dans l'aigu qui accompagnent l'arrivée de l'hélicoptère et correspondent à la tension due à la densification de la figure du prédateur.

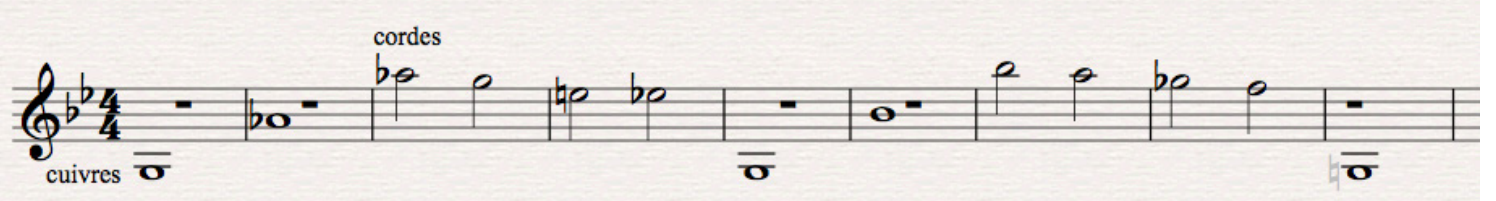

Figure 9: Harry Gregson-Williams, Total Recall, scène de poursuite en voiture, stingers aux cuivres et motifs descendants aux cordes dans l'aigu. Transcription personnelle à partir du film (00:53:18-00:53:32).

Lori manifeste son entrée au sein de la poursuite par la collision de son véhicule contre celui de la proie, accentuée d'un stinger (toujours sur un sol grave aux cuivres), puis d'un retour au plan intérieur du véhicule de la proie. L'identification de Lori par Douglas donne lieu à une tension soutenue par une nappe aux cordes (sur mi dans l'aigu). Alors que l'ensemble des éléments inhérents aux différentes entités prenant part à la poursuite sont introduits, une nouvelle dynamique survient et figure un premier ostinato (audible) aux cordes (déjà entendu lors de la scène se déroulant à Rekall). La structure de l'ostinato accuse les moments critiques de la tension narrative, 
présentant une expansion de certains motifs à l'octave, notamment lorsque la vitre arrière du véhicule de la proie éclate sous les tirs du soldat de l'UFB, lorsque le véhicule de la proie percute le mur du tunnel, et lorsque Melina freine pour heurter le véhicule du prédateur.

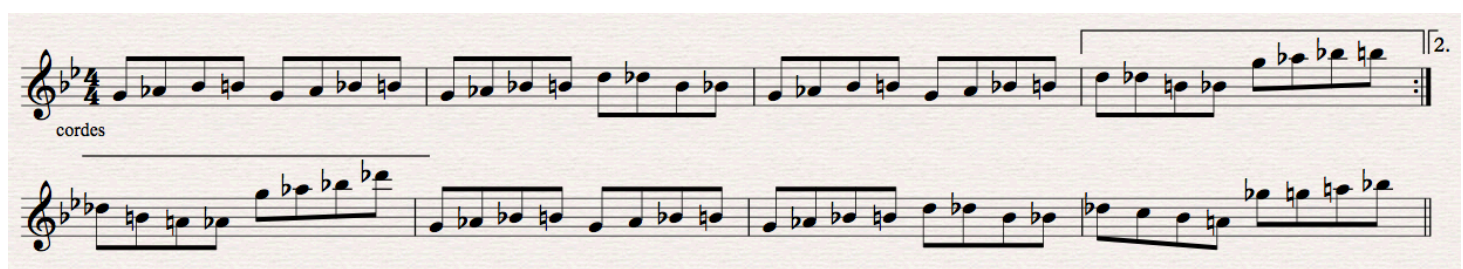

Figure 10: Harry Gregson-Williams, Total Recall, scène de poursuite en voiture, premier ostinato aux cordes. Transcription personnelle à partir du film (00:53:44-00:54:02).

Toute cette section en ostinato où la proie est criblée de tirs s'accompagne d'une répétition accentuée de la tonique et d'un glissement chromatique de sons synthétiques (du sol grave à l'octave), jusqu'à la collision entre l'arrière du véhicule de la proie et celui du soldat tireur. La mort du soldat, éjecté du véhicule, donne lieu à une montée du stinger aux cuivres (de sol à sib grave), un nouveau motif rythmique aux percussions, et une descente aux cordes reprise à l'aigu.

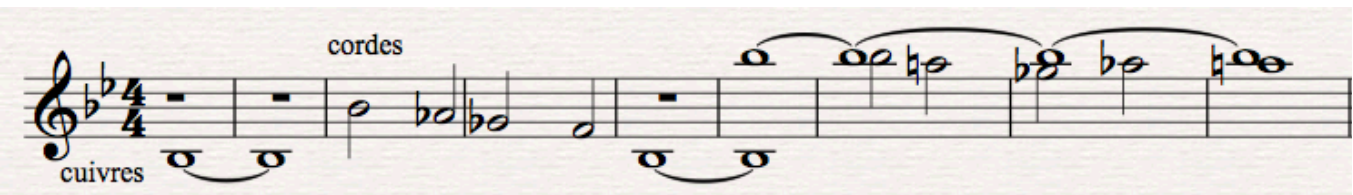

Figure 11 : Harry Gregson-Williams, Total Recall, scène de poursuite en voiture, stingers aux cuivres (sur sib grave) et deuxième motif de descente aux cordes. Transcription personnelle à partir du film (00:54:07-00:54:23).

La riposte de la proie marque un autre changement du dynamisme de la poursuite où elle se trouve désormais cernée par les véhicules du prédateur. Alors que Lori heurte le véhicule de Melina et Douglas, un nouvel ostinato aux cordes et un motif aux cuivres (récurrent d'ailleurs dans le film, par exemple lors de la poursuite dans les ascenseurs) signalent l'état critique de la situation de la proie.

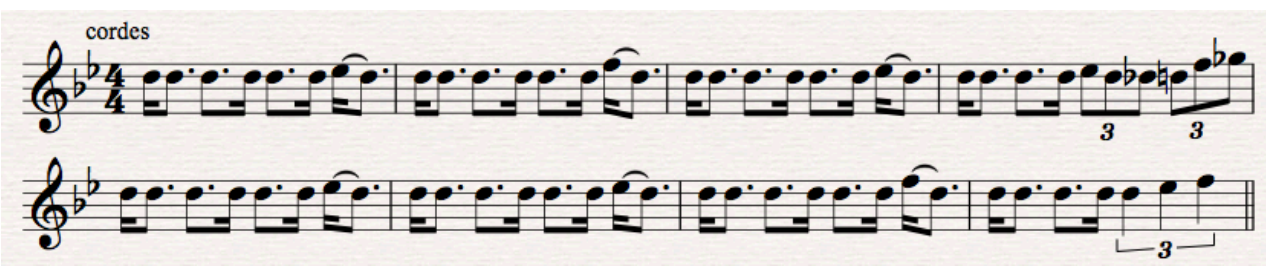

Figure 12 : Harry Gregson-Williams, Total Recall, scène de poursuite en voiture, deuxième ostinato aux cordes. Transcription personnelle à partir du film (00:54:24-00:54:36).

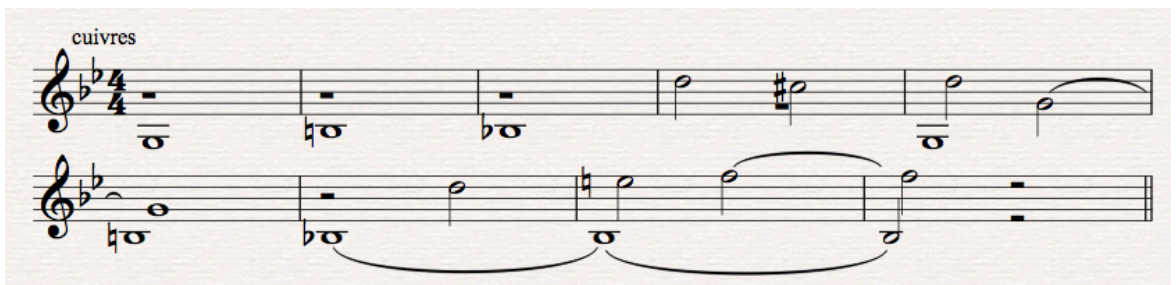

Figure 13 : Harry Gregson-Williams, Total Recall, scène de poursuite en voiture, motif aux cuivres qui accompagne l'ostinato de la figure 12. Transcription personnelle à partir du film (00:54:24-00:54:37). 
Le motif en ostinato aux cordes monte sur $f a$ alors que Douglas prend le volant. La suite du motif aux cuivres appuie sur sib grave, puis $f a$, en dissonance avec les réponses aux cuivres à l'octave. Le dernier mi aux cuivres dans l'aigu marque un point de climax, suivi de la suspension de l'ensemble des éléments sonores pour mettre en valeur les tirs de Melina sur le véhicule du prédateur. Une suspension temporelle survient ensuite caractérisée par le retrait de l'ensemble des éléments musicaux et un retour aux sons de l'environnement sonore de la route aérienne et du carambolage ${ }^{26}$. Elle a pour fonction de délimiter la fin de la première partie de la poursuite. Un crescendo en dissonance aux cuivres (sur la grave) introduit la deuxième partie de la poursuite, de même qu'un accent de sons synthétiques (sur ré).

\section{Développement - Deuxième partie}

Extrait vidéo 13 : Len Wiseman, Total Recall, scène de poursuite en voiture (2012), 00:55:07-00:58:11 (c) Sony Pictures.

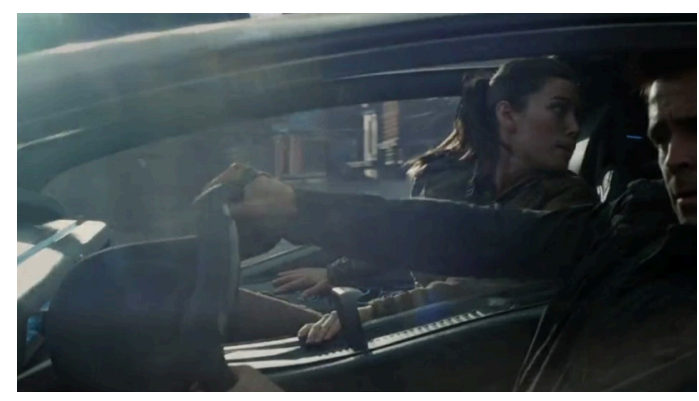

La deuxième partie du développement de la poursuite figure une tension croissante vers une situation critique où la proie, cernée, réussit à s'échapper. La descente au niveau inférieur et inversé de la route aérienne annonce le motif d'un nouvel ostinato aux cordes et donne la tonalité de ré mineur. L'ostinato est introduit avec la reprise de la fuite de la proie, et connaît une nouvelle forme suite à l'arrivée de Lori qui sera récurrente jusqu'au terme de la poursuite.

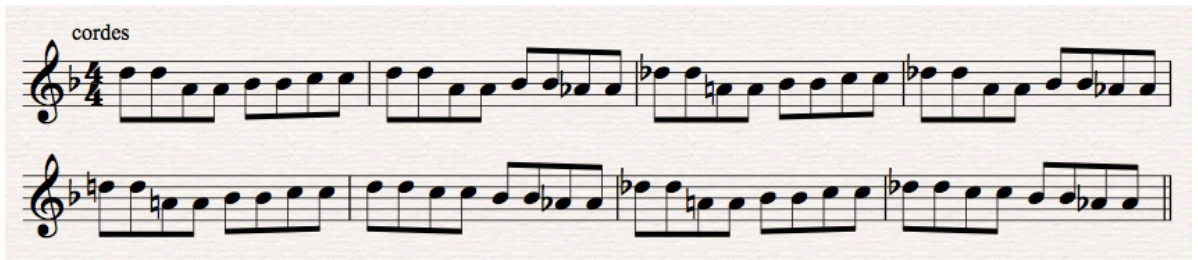

Figure 14 : Harry Gregson-Williams, Total Recall, scène de poursuite en voiture, ostinato principal de la deuxième partie du développement de la poursuite. Transcription personnelle à partir du film (00:55:46-00:55:57).

Lori donne l'ordre d'intercepter la proie (sur mi aux cuivres), puis tous les véhicules du prédateur sont appelés à intervenir (montée sur fa aux cuivres). L'entrée du motif répété des cordes et des cuivres (sur fa dans l'aigu) accompagne l'ordre de la centrale : "Establish barricade in zone 217 », et le retour sur le plan du véhicule de la proie. 


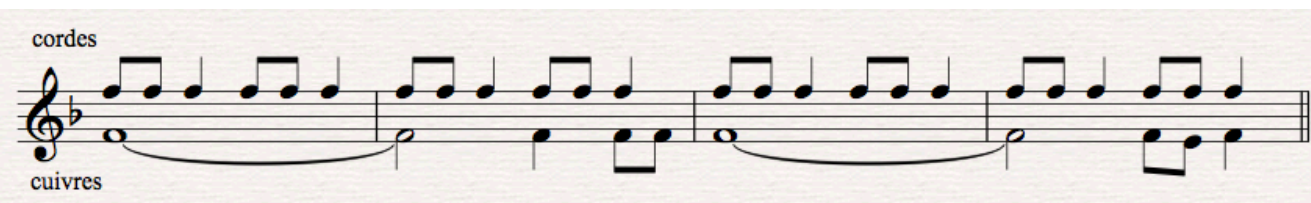

Figure 15 : Harry Gregson-Williams, Total Recall, scène de poursuite en voiture, motif aux cordes et aux cuivres qui vient densifier l'ostinato de la figure 14. Transcription personnelle à partir du film (00:55:57-00:56:02).

Un deuxième ordre de Lori marque l'entrée d'un nouvel ostinato aux cordes. De même, les cordes en dissonance dans l'aigu (sur ré, puis $f a$ aigu) sont accentuées lorsque Lori arrive à la hauteur de Melina et Douglas. Les véhicules du prédateur forment une barricade pour intercepter la proie, aveuglée par l'hélicoptère. L'entrée d'un long motif aux cuivres intervient alors avec une montée de l'ostinato des cordes dans l'aigu (ce motif revient d'ailleurs au cours de la poursuite dans les ascenseurs). La musique instaure progressivement une densification de l'ensemble sonore et une superposition de plusieurs motifs rythmiques. De même, des échanges aux cuivres entre des registres opposés mettent en valeur la montée chromatique aux cors (sib-dodo\#). Enfin, l'entrée des trompettes (sur fa, puis sur la dans l'aigu) accompagne l'affirmation de Lori : "You are clear to engage ». La proie se trouve en situation critique, accentuée par la densité des éléments sonores, la diversité des motifs rythmiques, l'étendue des registres, et l'ampleur de l'effectif instrumental, et ce jusqu'à la suspension temporelle et le climax final en crescendo.

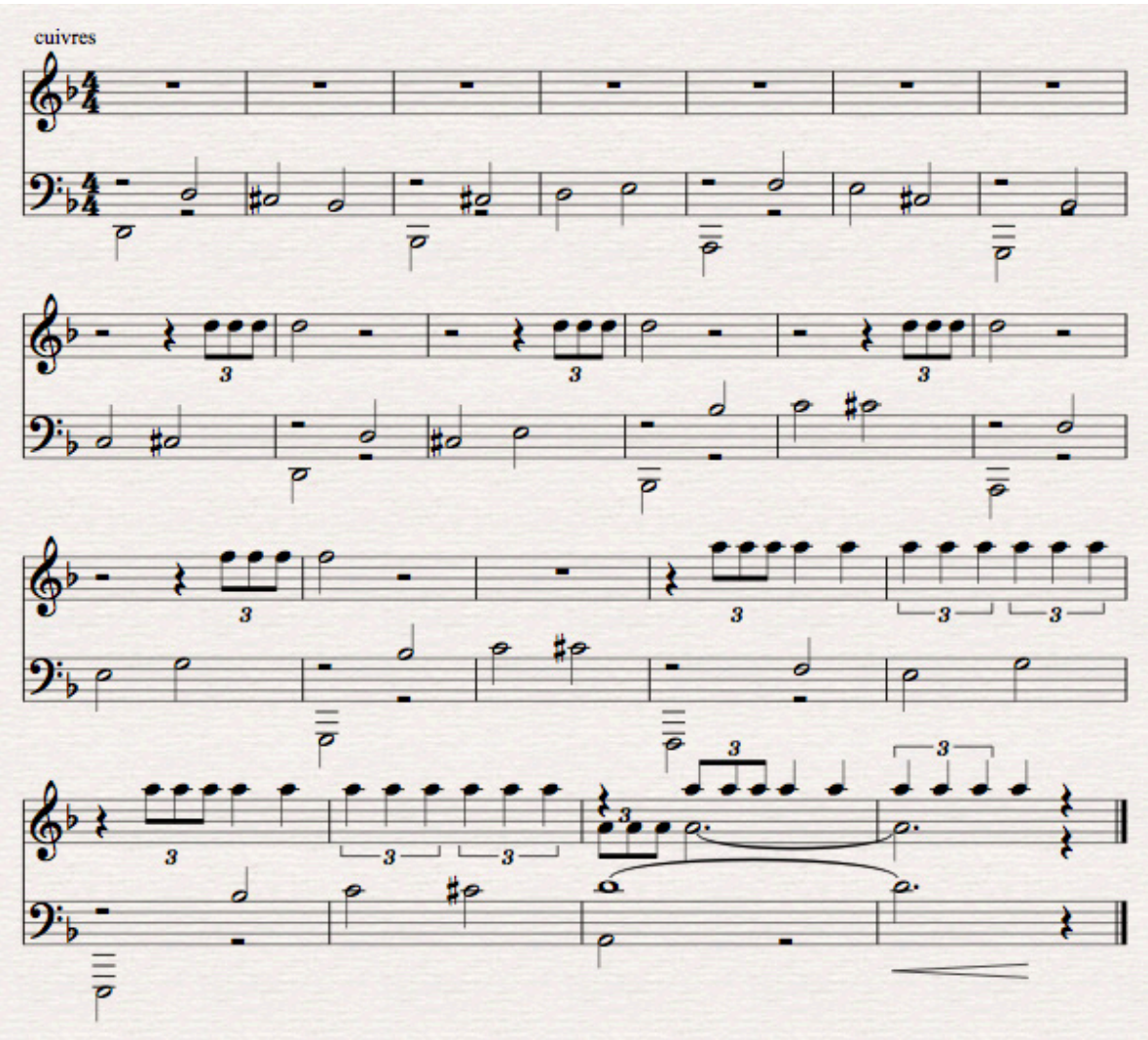

Figure 16: Harry Gregson-Williams, Total Recall, scène de poursuite en voiture, principaux motifs aux cuivres à partir de l'arrivée de l'hélicoptère devant la barricade des véhicules de la police de l'UFB. Ces motifs évoluent sur l'ostinato de la figure 14 et les motifs de la figure 15. Transcription personnelle à partir du film (00:56:30-00:56:59). 


\section{Résolution}

La résolution de la poursuite survient avec une suspension temporelle au moment de la chute du véhicule de la proie qui s'échappe par le moyen d'une démagnétisation de la route aérienne. Seuls le son de l'air et le souffle des protagonistes demeurent tout au long de la chute. Le silence suspend la tension et renforce l'effet de collision lorsque le véhicule percute le sol. L'écrasement donne ensuite lieu à des nappes de cordes (sur un si grave) et à un plan rapproché sur Douglas, puis sur Melina, inconsciente, dont la tête a heurté la vitre. Le son des sirènes de police au loin indique l'aspect non conclusif de la poursuite, alors que Douglas prend le pouls de Melina (sur mi aigu). Douglas sort du véhicule, soutenu par la montée du mi sur fa\# et l'accord de si mineur. Au point de vue narratif, la poursuite aura permis la rencontre de Douglas et Melina, de même qu'un renforcement de l'identification aux protagonistes.

Tout au long de la poursuite, et de façon notable au cours de la première partie, la tonique est employée pour structurer les différents épisodes de l'action et les changements de dynamique entre les plans d'ensemble et les plans resserrés à l'intérieur du véhicule de la proie. De même, si la reprise d'un motif à l'octave est employée à des fins de densification et de mise en tension, on peut également lui attribuer une fonction méliorative pour assurer la perception des ostinati malgré la densification de l'ensemble sonore. On retrouve une mesure à quatre temps, le recours à des motifs cycliques, une combinaison de multiples sources sonores, et l'intervention de phrases larges aux cuivres. En outre, la partie orchestrale est complétée par divers samples qui soulignent les moments critiques de la narration, par exemple lorsque le soldat est éjecté du véhicule, ou pour accompagner les ordres adressés par Melina. La deuxième partie de la poursuite présente une croissance de la tension au fil des ordres énoncés par le prédateur, disposant la proie dans une situation de plus en plus critique, et donnant la primauté à la sensation en opposition aux réflexions émises par les dialogues au cours de la première partie. De multiples ostinati et des variations brusques et serrées ponctuent la partie finale de la poursuite. L'ensemble de la séquence procède selon une logique d'accumulation, de densification et d'élévation vers l'aigu, notamment dans sa structure harmonique, passant de la tonalité de sol mineur à celle de ré mineur en deuxième partie. Enfin, on peut noter un recours à certaines notes isolées, ou nappes de cordes, pour teinter au même titre qu'un effet sonore certaines tensions, notamment au cours des dialogues.

\section{CONCLUSION}

$\mathrm{Au}$ sein du régime d'effets spectaculaires et de variations rythmiques instaurés par le montage visuel, la fonction de la musique dans les scènes de poursuite dépasse résolument le simple cadre de l'accompagnement de l'image. Ainsi, par le recours à différentes techniques compositionnelles parfois influencées par d'autres domaines de la création musicale contemporaine, l'ensemble sonore de la poursuite répond d'une part à une fonction structurante à la fois pour consolider les différentes phases de la scène et raffermir l'identité des éléments narratifs qui y participent. D'autre part, la 
musique répond à une fonction immersive et influence l'engagement spectatoriel non seulement dans la narrativité filmique, mais également dans le cinéma comme médium de l'expression du mouvement et comme " cinéma d'effets " (Gunning 2006, p. 65). En réponse à ces fonctions, l'esthétique sonore et le mode de production musicale promues par Remote Control s'avèrent particulièrement efficaces par l'importance accordée au travail sur la texture sonore et le rythme, et la forte homogénéité entre les différents éléments de l'ensemble sonique du film ; une composition musicale dont la logique est centrée sur l'effet, de même que sur la corrélation et la mise en valeur des autres éléments de la sémiotique filmique. Enfin, l'esthétique sonore des scènes étudiées ne constitue pas, à notre avis, un renversement des pratiques musicales du néoclassicisme hollywoodien, mais bien un prolongement de celles-ci. Dans la foulée de l'analyse visuelle de Bordwell (Bordwell 2002), Jeff Smith (Smith 2013) transpose à la dimension sonore des films la thèse de 1' "intensified continuity", et soutient que le son des productions hollywoodiennes contemporaines fonctionne

either by enhancing the immersive quality of the intensified continuity style or by heightening the visceral quality of particular storytelling devices. Yet even as intensified continuity sound serves to amplify, quite literally, the sensational qualities of contemporary Hollywood cinema, it remains firmly anchored within the principles of unity, clarity, and linearity that still govern the transmission of narrative information to viewers (Smith 2013, p. 347).

Qu'il s'agisse du recours à l'ostinato ou à la montée dans l'aigu pour illustrer la tension, ou encore de la dynamisation de la poursuite grâce à la suspension temporelle, plusieurs techniques compositionnelles abordées lors de notre étude trouvent leur source dans des pratiques musicales antérieures qui ont fait l'objet d'une mutation corrélée à l'évolution des technologies et des approches compositionnelles du cinéma hollywoodien contemporain. Ainsi, il semble que le portrait dressé de l'esthétique musicale des scènes de poursuite réalisées par Remote Control s'inscrive, au même titre que le style visuel des films américains contemporains, dans une logique de continuité et d'intensification des techniques établies, en concordance avec les propositions de Bordwell et Smith.

\section{BIBLIOGRAPHIE}

Allyn, Conor (2013), Java Heat, DVD, Metropole Films, 750265437.

Altman, Rick (2013), «Establishing sound », Cinémas, vol. 24, nº 1, p. 19-33.

Arijon, Daniel ([1976] 2004), La grammaire du langage filmé, traduit de l'anglais par Évelyne Bonnet, Paris, Éditions Dujarric.

Audissino, Emilio (2014), John Williams's Film Music. Jaws, Star Wars, Raiders of the Lost Ark, and the Return of the Classical Hollywood Music Style, Madison, The University of Wisconsin Press.

Auerbach, Jonathan (2000), "Chasing Film Narrative. Repetition, Recursion, and the Body in Early Cinema », Critical Inquiry, vol. 26, n 4, p. 789-820.

Barker, Jennifer (2009), The Tactile Eye. Touch and the Cinematic Experience, Berkeley, University of California Press.

Balfe, Lorne (2017), Geostorm. Original Motion Picture Soundtrack, WaterTower Music, piste 6, «Lightning Chase ». 
Bordwell, David (2002), "Intensified Continuity Visual Style in Contemporary American Film », Film Quarterly, vol. 55, $\mathrm{n}^{\circ} 3$, p. 16-28.

Bordwell, David (2006), The Way Hollywood Tells It, Berkeley, University of California Press.

Burch, Noël (1983), « Passion, poursuite. La linéarisation », Communications, nº 38, p. 30-50.

Butler, Mark J. (2006), Unlocking the Groove. Rhythm, Meter, and Musical Design in Electronic Dance Music, Bloomington, Indiana University Press.

Calleja, Gordon (2007), « Revising Immersion. A Conceptual Model for the Analysis of Digital Game Involvment ", Proceedings of Situated Play, DiGRA Conference, Tokyo, Université de Tokyo, p. 83-90.

Chion, Michel (1990), L'Audio-vision. Son et image au cinéma, Paris, Armand Colin.

Donnelly, Kevin J. (2005), "Demonic Possession. Horror Film Music », dans Kevin J. Donnelly, The Spectre of Sound. Music in Film and Television, London, BFI, p, 88-109.

Donnelly, Kevin J. (2009), "Saw Heard. Musical Sound Design in Contemporary Cinema ", dans Warren Buckland (dir.), Film Theory and Contemporary Hollywood Movies, New York/London, Routledge, 2009, p. 103-123.

Donnelly, Kevin J. (2010), « Hearing Deep Seated Fears. John Carpenter's The Fog (1980) ", dans Neil Lerner (dir.), Music in the Horror Film. Listening to Fear, New York/London, Routledge, 2010, p. 152-167.

Green, David (2016), Teenage Mutant Ninja Turtles. Out of the Shadows, DVD, Paramount, 59180521000.

Greengrass, Paul ([2009] 2010), Green Zone, Blu-ray, Studio Canal, EDV 1392/827630-8.

Gregson-Williams, Harry (2012a), Total Recall. Original Motion Picture Soundtrack, Madison Gate Records, piste 6 « Rooftop Chase».

Gregson-Williams, Harry (2012b), Total Recall. Original Motion Picture Soundtrack, Madison Gate Records, piste 10 « Car Chase, Pt. 1 ».

Gregson-Williams, Harry (2012c), Total Recall. Original Motion Picture Soundtrack, Madison Gate Records, piste 11 "Car Chase, Pt. 2 ».

Grimshaw, Mark (2012), "Sound and Player Immersion in Digital Games », dans Trevor Pinch et Karin Bijsterveld (dir.), The Oxford Handbook of Sound Studies, New York, Oxford University Press, p. 347-366.

Gunning, Tom ([1986] 2006), «Le cinéma d'attration. Le film des premiers temps, son spectateur, et l'avant-garde ", traduit de l'anglais par Franck Le Gac, 1895. Mille huit cent quatre-vingt-quinze, $\mathrm{n}^{\circ}$ 50, p. $55-65$.

Hexel, Vasco (2016), Hans Zimmer and James Newton Howard's The Dark Knight. A Film Score Guide, London, Rowman \& Littlefield.

Lerner, Neil (2010), Music in the Horror Film. Listening to Fear, New York/London, Routledge.

McCaffrey, Donald W. (2016), "The Evolution of the Chase in the Silent Screen Comedy ", dans Barry Keith et Malisa Kurtz, Notions of Genre. Writings on Popular Film before Genre Theory, Austin, University of Texas Press, p. 60-69.

McMahan, Alison (2003), «Immersion, Engagement, and Presence. A New Method for Analyzing 3-D Video Games », dans Mark J.P. Wolf et Bernard Perron (dir.), The Video Game Theory Reader, New York, Routledge, 2003, p. 67-87.

Miller, George (2015), Mad Max. Fury Road, DVD, Warner Home Video, 3000065151.

Minett, Mark (2013), "Beyond the Badass. Electronic Dance Music Meets Film Music Practice», New Review of Film and Television Studies, vol. 11, n 2, p. 191-210.

Mothersbaugh, Mark (2017), Thor: Ragnarok. Original Motion Picture Soundtrack, Hollywood Records, piste 18 "Sakaar Chase ».

Rodgers, Tara (2003), "On the Process and Aesthetics of Sampling in Electronic Music Production », Organized Sound, vol. 8, n 3, p. 313-320. 
Shilling, Russell, Michael Zyda et E. Casey Wardynski (2002), «Introducing Emotion into Military Simulation and Videogame Design: America's Army. Operations and VIRTE», Presence. Teleoperators and Virtual Environments, vol. 11, n ${ }^{\circ}$, p. 435-439.

Stilwell, Robynn J. (2007), «The Fantastical Gap Between Diegetic and Nondiegetic », dans Daniel Goldmark, Lucy Kramer et Richard Leppert (dir.), Beyond the Soundtrack. Representing Music in the Cinema, Berkeley, University of California Press, p. 184-202.

Smith, Jeff (2013), «The Sound of Intensified Continuity », dans John Richardson, Claudia Gorbman, Carol Vernallis (éd.), The Oxford Handbook of New Audiovisual Aesthetics, New York, Oxford University Press, p. 331-356.

Turtletaub, Jon (2010), The Sorcerer's Apprentice, DVD, Disney DVD, 105672/02.

Waititi, Taika (2017), Thor. Ragnarok, DVD, Marvel Studios, 147600/02.

Wescott, Steven D. (1989), « Miklós Rózsa's Ben Hur. The Musical Dramatic Function of the Hollywood Leitmotiv », dans Clifford McCarty (dir.), Film Music I, New York, Garland Publishing, p. 183-207.

Wiseman, Len (2012), Total Recall, DVD, Sony Pictures Home Entertainment, 40960.

Zimmer, Hans (s.d.), Hans-Zimmer.com, http://www.hans-zimmer.com/index.php, consulté le 12 mars 2018. 


\section{AnNeXe 1}

\begin{tabular}{|c|c|c|c|c|c|}
\hline Titre & Sortie & Réalisateur(s) & Compositeur(s) & Pistes (Durée) & Séquences filmiques correspondantes (ca.) \\
\hline The Sorcerer's Apprentice & 2010 & $\begin{array}{l}\text { TURTELTAUB, } \\
\text { Jon }\end{array}$ & RABIN, Trevor & 14. Car Chase $(3: 54)$ & Car Chase $(01: 12: 41-01: 16: 33)$ \\
\hline Green Zone & 2010 & $\begin{array}{l}\text { GREENGRASS, } \\
\text { Paul }\end{array}$ & POWELL, John & 12. Attack and Chase $(5: 25)$ & Attack and Chase (01:29:04-01:36:51) \\
\hline Total Recall & 2012 & WISEMAN, Len & $\begin{array}{l}\text { GREGSON-WILLIAMS, } \\
\text { Harry }\end{array}$ & $\begin{array}{l}\text { 6. Rooftop Chase }(2: 23) \text {; } \\
\text { 10. Car Chase Pt. } 1(2: 44) \text {; } \\
\text { 11. Car Chase Pt. } 2(1: 34) \text {; } \\
\text { 14. Elevator Chase }(5: 21)\end{array}$ & $\begin{array}{l}\text { Rooftop Chase (00:37:09-00:39:50) } \\
\text { Car chase Pt. } 1 \& 2(00: 52: 28-00: 57: 01) \\
\text { Elevator Chase }(01: 16: 12-01: 21: 06)\end{array}$ \\
\hline Java Heat & 2013 & ALLYN, Conor & BURNETT, Justin Caine & 15. Motorcycle Chase $(2: 49)$ & Motorcycle Chase (00:45:20-00:48:00) \\
\hline Drive Hard & 2014 & $\begin{array}{l}\text { TRENCHARD- } \\
\text { SMITH, Brian }\end{array}$ & JACOBS, Bryce & 7. SUV Chase $(1: 29)$ & SUV Chase $(00: 14: 45-00: 18: 50)$ \\
\hline $\begin{array}{l}\text { Transformers: Tbe Age of } \\
\text { Extinction }\end{array}$ & 2014 & BAY, Michael & JABLONSKY, Steve & 16. Hong Kong Chase (1:45) & Hong Kong Chase (01:58:33- $02: 00: 22)$ \\
\hline Mad Max: Fury Road & 2015 & MILLER, George & $\begin{array}{l}\text { JUNKIE XL (as Tom } \\
\text { Holkenborg) }\end{array}$ & N.A. & $(01: 29: 05-01: 46: 56)$ \\
\hline $\begin{array}{l}\text { Run All Nigbt [Nigbt } \\
\text { Run] }\end{array}$ & 2015 & $\begin{array}{l}\text { COLLET-SERRA, } \\
\text { Jaume }\end{array}$ & $\begin{array}{l}\text { JUNKIE XL (as Tom } \\
\text { Holkenborg) }\end{array}$ & 8. Cop Chase $(5: 10)$ & Cop Chase $(00: 36: 29-00: 41: 06)$ \\
\hline $\begin{array}{l}\text { Teenage Mutant Ninja } \\
\text { Turtles: Out of the } \\
\text { Sbadows } \\
\text { [Ninja Turtles 2] }\end{array}$ & 2016 & GREEN, David & JABLONSKY, Steve & $\begin{array}{l}\text { 5. Shredder Escape }(6: 27) \text {; } \\
\text { 9. Foot Clan Chase }(3: 05)\end{array}$ & $\begin{array}{l}\text { Shredder Escape }(00: 16: 53-00: 23: 11) \\
\text { Foot Clan Chase }(00: 37: 11-00: 40: 05)\end{array}$ \\
\hline Jason Bosirne & 2016 & $\begin{array}{l}\text { GREENGRASS, } \\
\text { Paul }\end{array}$ & $\begin{array}{l}\text { BUCKLEY, David; } \\
\text { POWELL, John }\end{array}$ & $\begin{array}{l}\text { 4. Motorcyle Chase }(6: 53) \text {; } \\
\text { 13. Strip Chase }(4: 59)\end{array}$ & $\begin{array}{l}\text { Motorcycle Chase }(00: 19: 36-00: 32: 59) \\
\text { Strip Chase }(01: 38: 48-01: 45: 16)\end{array}$ \\
\hline Geostorm & 2017 & DEVLIN, Dean & BALFE, Lorne & 6. Lightning Chase $(5: 07)$ & Lightning Chase (01:15:45-01:21:51) \\
\hline The Hitman's Bodyguard & 2017 & HUGHES, Patrick & ÖRVARSSON, Atli & 8. Amsterdam Chase $(3: 25)$ & Amsterdam Chase (01:10:00-01:16:32) \\
\hline $\begin{array}{l}\text { Transformers: The Last } \\
\text { Knight }\end{array}$ & 2017 & BAY, Michael & JABLONSKY, Steve & 12. Drone Chase ( $5: 07)$ & Drone Chase (00:52:50-00:58:20) \\
\hline Tbor: Ragnarok & 2017 & WAITTTI, Taika & MOTHERSBAUGH, Mark & 18. Sakaar Chase $(2: 12)$ & Sakaar Chase (01:31:30-01:35:15) \\
\hline
\end{tabular}

Tableau des pistes d'albums de film d'action hollywoodiens comportant le terme "chase " et les séquences filmiques correspondantes. 